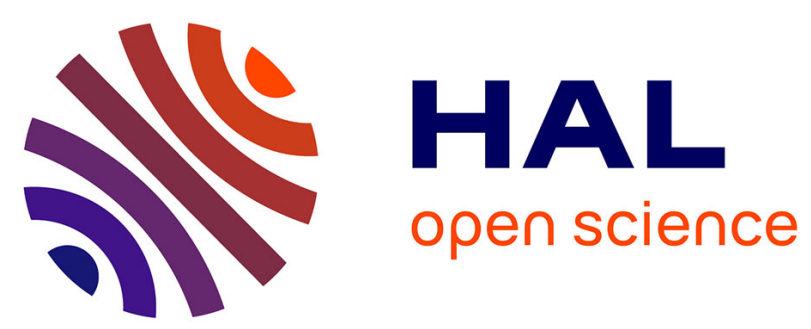

\title{
PANTOGRAPHIC LATTICES WITH NON-ORTHOGONAL FIBRES: EXPERIMENTS AND THEIR NUMERICAL SIMULATIONS
}

Emilio Turco, Maciej Golaszewski, Ivan Giorgio, Francesco D 'Annibale

\section{- To cite this version:}

Emilio Turco, Maciej Golaszewski, Ivan Giorgio, Francesco D 'Annibale. PANTOGRAPHIC LATTICES WITH NON-ORTHOGONAL FIBRES: EXPERIMENTS AND THEIR NUMERICAL SIMULATIONS. 2017. hal-01443679

\author{
HAL Id: hal-01443679 \\ https://hal.science/hal-01443679
}

Preprint submitted on 23 Jan 2017

HAL is a multi-disciplinary open access archive for the deposit and dissemination of scientific research documents, whether they are published or not. The documents may come from teaching and research institutions in France or abroad, or from public or private research centers.
L'archive ouverte pluridisciplinaire HAL, est destinée au dépôt et à la diffusion de documents scientifiques de niveau recherche, publiés ou non, émanant des établissements d'enseignement et de recherche français ou étrangers, des laboratoires publics ou privés. 


\title{
PANTOGRAPHIC LATTICES WITH NON-ORTHOGONAL FIBRES: EXPERIMENTS AND THEIR NUMERICAL SIMULATIONS
}

\author{
EMILIO TURCO, MACIEJ GOLASZEWSKI, IVAN GIORGIO, AND FRANCESCO D'ANNIBALE
}

\begin{abstract}
Current research in metamaterials design is pushing to fill the gap between mathematical modelling and technological applications. To meet these requirements predictive and computationally effective numerical tools needs to be conceived and applied. In this paper we describe the performances of a naturally discrete model [1] and those of a second gradient continuum model [2] for pantographic structures with non-orthogonal fibres comparing them with some experimental results. The interest in these structures resides in the exotic behaviour that they have already shown [3] and their study seems promising. The comparison which we present here shows that, depending on the length scale characterising the structural cell of pantographic sheets, either discrete or continuum model performance and/or behaviour may prevail. Some homogenization interesting problems are listed in the conclusions in the hope that they may be rigorously studied with the most advanced mathematical tools.
\end{abstract}

\section{INTRODUCTION}

Current research in metamaterials design is pushing to fill the gap between mathematical modelling and technological applications. Although both evolutionary selection in living organism and the past engineering scientifically based research have already promoted of "exotic" metamaterials (the bone tissue is one example while woven fabrics gives another one) it is only a recent issue the systematics research of tailored materials having fixed (well-determined a priori) uses and applications.

To meet all the requirements imposed by determined and well-specified applications it is needed to establish a designing procedure which involve the important step concerning the development of some predictive and computationally effective numerical tools. These tools will be then used to verify experimental measurements output and subsequently to design specifically adapted materials.

In this paper we focus on a specific, but in our opinion relevant, task: to formulate and to compare the performances of a discrete versus a continuum model for pantographic lattices, sometimes also called pantographic sheets.

The interest in these structures resides in the exotic behaviour that they have shown $[2,3]$ and their study seems promising. In particular pantographic structures:

- are the actual realisation of a (often disputed) continuum model: i.e. second gradient materials; indeed pantographic sheets are one of the first mechanical structures which have been proven $[4,5]$ to need a second gradient models at a given macroscopic length-scale;

- have been proven to have very promising properties in wave propagation, representing an example of effective wave-guides [6];

- have shown promising toughness properties, which suggest that they could be fruitfully embedded in novel composite materials.

The comparison between continuum versus discrete models which we present here shows that depending on the length scale characterising the structural cell of pantographic sheets some mechanical properties may prevail. Moreover the performances, in a given situation, of one of the two models may be more satisfactory than the other one, and this may happen independently of the real physical structure of the pantographic lattice depending only on the numerical properties of considered codes. We remark that some homogenization interesting problems are now to be faced, in order to give an effective and reliable mathematical basis to the presented results. 

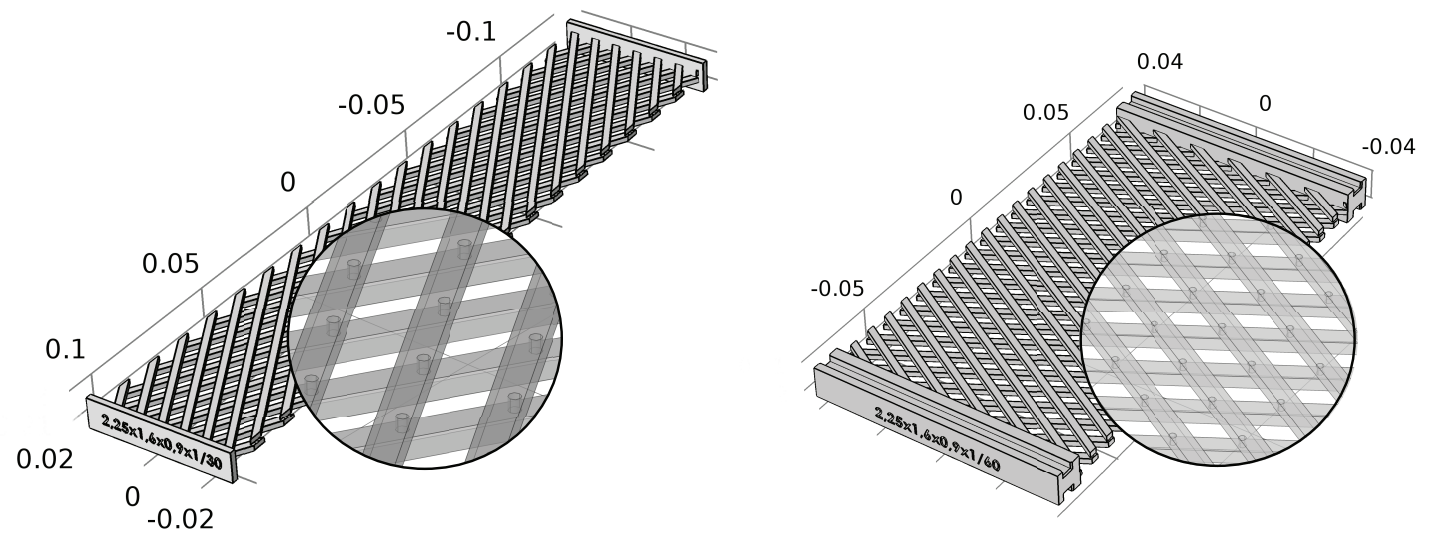

FIGURE 1. Three-dimensional drawing of pantographic lattice fabrics.

The paper, after this brief introduction, is organised in accordance with the following plan. In Section 2 there is a brief discussion relative to two simple displacement-controlled traction tests: their main difference consists in the different orientation of the fibres. In Section 3 are described two models which are strictly correlated: indeed the first one, discrete model, is the meso-mechanical basis of the second one, continuum model. From these models, in Section 4, the numerical simulations relative to the two experiments are presented and discussed thoroughly highlighting the closeness of the simulations to the experiments. Finally, in Section 5 there are some concluding remarks which anticipate future perspectives and challenges.

\section{Some EXPERIMENTAL EVIDENCES}

We consider the two fabrics depicted in Figure 1. Both of them are pantographic structures formed by two arrays of beams with rectangular cross-section connected by means of cylindrical pivots in their intersection points.

A more technical representation is reported in Figures 2 and 3 for the fabric on the left and on the right of Figure 1 respectively. It is evident that the two fabrics have exactly the same fibres (cross-section ad length) but arranged in a different way (in both cases the fibres are non-orthogonal): the angle between them is $\varphi=120^{\circ}$ in the first case, see Figure 2, and $\varphi=60^{\circ}$ in the second one, see Figure 3.

In some recent papers, see [7, 8, 9] similar structures, but with orthogonal fibres, have been intensively studied from the experimental point of view and their results compared with the predictions of some numerical simulations deriving from a generalised Hencky-type model and from a second gradient continuum model, see [2].

The fabrics sketched in Figures 2 and 3 were built by using the 3D printing technology, in polyamide (PA 2200) by a SLS Formiga P100. For this material the Young's modulus was estimated between 1.51.7 GPa following the rules of EN ISO 527 and EN ISO 178.

Both the fabrics have been tested by means of a traction test: the left side is clamped and on the right side is assigned a constant displacement $u$ parallel to the greater sides, from zero to $u_{\max }$, by using the MTS Bionix system strength machine selecting a velocity of about $5 \mathrm{~mm} / \mathrm{min}$.

The first experiment concerns the fabric reported in Fig. 2. In this case the imposed maximum displacement is $u_{\max }=23.7 \mathrm{~mm}$ and the three pictures reported in Fig. 4 show the initial, the intermediate and the final configuration. 


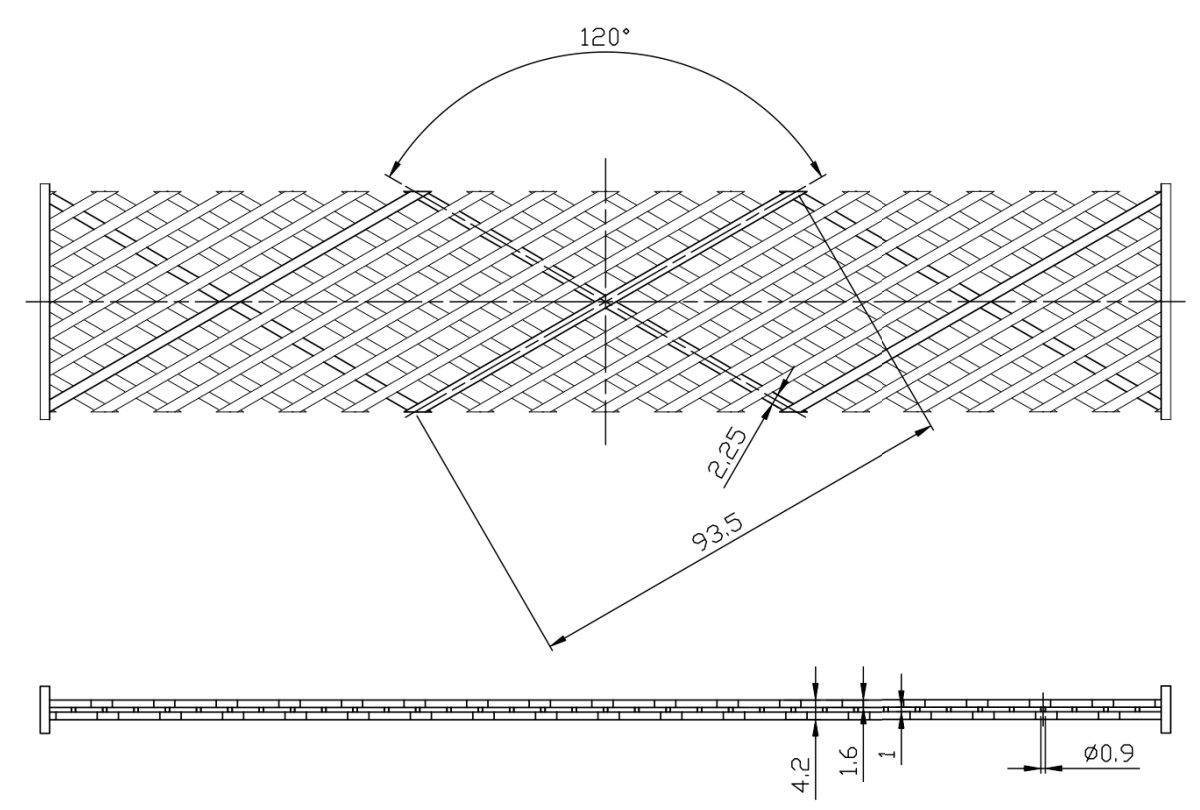

FIGURE 2. Drawing of a pantographic lattice with non-orthogonal fibers $\left(\varphi=120^{\circ}\right)$.

Figure 5 reports the global structural reaction of the right side of the fabric (in the direction of the greater sides) as the non-dimensional displacement parameter $u / L_{1}$ increases being $L_{1}$ the length of the greater sides of the fabric.

The second test concerns the fabric depicted in Fig. 3 which is made by the same polyamide of the previous test and of the same fibres but, this time, arranged using the angle $\varphi=60^{\circ}$.

Also in this case the fabric has been tested by means of a simple traction test: an increasing assigned displacement $u$ on the right side (parallel to the greater sides) from zero to the value $u_{\max }=74.7 \mathrm{~mm}$. Three pictures have been taken during the loading process (the strength machine and the velocity of the test are unchanged), see Fig. 6, corresponding to the initial, the intermediate and the final configuration respectively.

As before, during the experimental test the global structural reaction $R$ on the right side (in the direction of the greater sides) has been measured. Figure 7 reports $R$ as the non-dimensional displacement parameter $u / L_{1}$ increases being $L_{1}$ the length of the greater side of the fabric.

Looking at Figs 5 and 7 we remark the different behaviour of the two fabrics built using exactly the same quantity of polyamide: the first one, $\varphi=120^{\circ}$, is much more stiff than the second one showing the relevance of the fibres orientation in the mechanical behaviour of this kind of metamaterial.

\section{DisCRETE AND CONTINUUM MODELS FOR PANTOGRAPHIC SHEETS}

In this Section we shortly describe a discrete Lagrangian model, or a generalized Hencky-type model (see [1]), and a second gradient continuum model, see [2], which we consider here to be possible models for planar pantographic structures. Their predictive performances will be analysed in the following section, here we limit ourselves to remark that the Lagrangian model and the second gradient continuum model are strictly connected since the first is the meso-mechanical basis of the second one (macro-model). In rough terms, the continuum model is obtained from a limiting process from the discrete Lagrangian model. For this reason, we present the basics, kinematics and strain energy, of the two models together starting, obviously, from the discrete Lagrangian (meso-mechanical) model.

3.1. Discrete and continuous kinematics. Starting from the observation of the physical model obtained by the $3 \mathrm{D}$ printing technology, see again Figure 1, we deduce that a basic model, already discrete since 


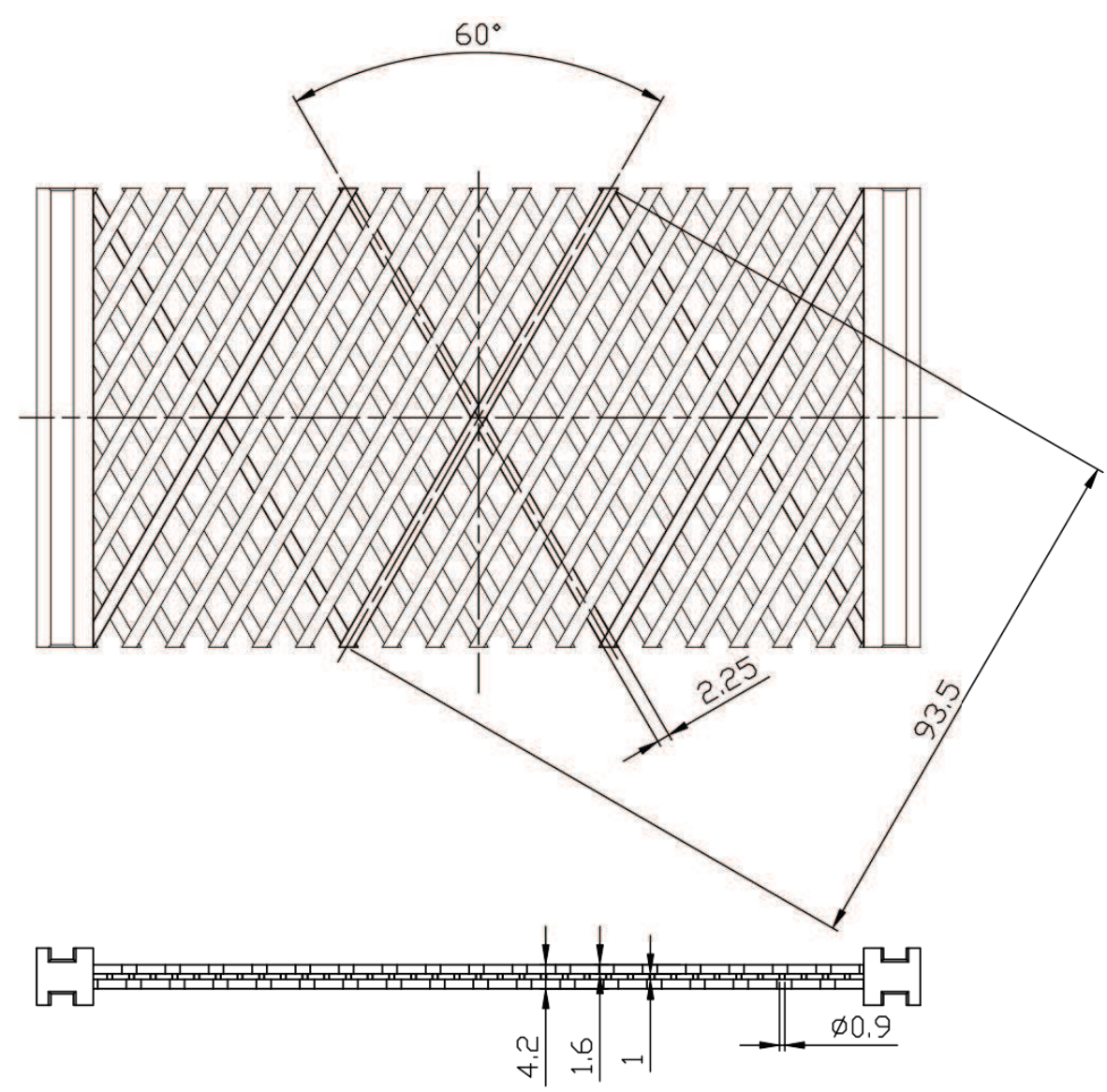

FiguRE 3. Drawing of a pantographic lattice with non-orthogonal fibres $\left(\varphi=60^{\circ}\right)$.

it is generated in the Hencky-vision, can be conceived by modelling the interconnecting pivots as nodes linked each other by means of extensional springs (pairwise interaction) and by means of rotational springs, both bending springs (triple interaction on a fibre) and shear springs (triple interaction on a fibre of the same array and also on the nearest pivots on the other direction). In this way, we can represent the pantographic sheet (schematically) as in Figure 8 by using the kinematical descriptors well-depicted in the sketch reported in Figure 9.

Discrete and continuum models deal with a finite or infinite configuration spaces, respectively. To be precise:

- the discrete model involves the introduction of a set of Lagrangian parameters specifying the position of all the material particles modelling the pivots: they are initially located in the nodes of the reference configuration and then displace to be located in the actual configuration. In planar motion a set of $2 N$ coordinates is sufficient (if $N$ is the number of considered nodes, the generic of which has referential position given $P_{i, j}$ ), such a set of Lagrangian coordinates could be given by the corresponding position $p_{i, j}$;

- the continuous model involves a regular field $\chi$ (at least $C^{0}$, its further regularity being specified by the postulated strain energy) defined in the reference configuration and mapping a generic material particle in its actual position.

Following Piola's Ansatz (see [10]) we may assume (see also [2]) that the correspondence between discrete and continuous model is given by assuming that the following equality holds:

$$
\chi\left(P_{i, j}\right)=p_{i, j}
$$


3.2. Discrete and continuous energies. Total energy, in hard devices induced deformations (and in absence of relevant volume forces) reduces to the strain energy only. Therefore, in both discrete and continuous models, it is the only kind of energy to be specified.

The postulated expression for the discrete Lagrangian strain energy, see $[2,1]$ for a comprehensive description, (in terms of the Lagrangian coordinates $p_{i, j}$ ) is given by:

$$
\begin{aligned}
W\left(\left\{p_{i, j}\right\}\right) & =\frac{1}{2} \sum_{i} \sum_{j} a_{i, j}^{\left(\alpha_{1}\right)}\left(\left\|p_{i+1, j}-p_{i, j}\right\|-\varepsilon\right)^{2}+\sum_{i} \sum_{j} a_{i, j}^{\left(\alpha_{2}\right)}\left(\left\|p_{i, j+1}-p_{i, j}\right\|-\varepsilon\right)^{2} \\
& +\sum_{i} \sum_{j} b_{i, j}^{\left(\alpha_{1}\right)}\left(\cos \beta_{i, j}^{\left(\alpha_{1}\right)}+1\right)+\sum_{i} \sum_{j} b_{i, j}^{\left(\alpha_{2}\right)}\left(\cos \beta_{i, j}^{(2)}+1\right) \\
& +\frac{1}{2} \sum_{i} \sum_{j} \sum_{q} s_{i, j}^{(q)}\left(\sigma_{i, j}^{(q)}-\bar{\sigma}_{i, j}^{(q)}\right)^{2},
\end{aligned}
$$

where the first two addends are related to the extensional energy of the fibres in $\alpha_{m}$-directions $(m=1,2$, $\varepsilon$ is the distance between to nearest pivots), see Figs. 8 and 9(a), being $a_{i, j}^{\left(\alpha_{m}\right)}$ the extensional stiffness parameters in $\alpha_{m}$-direction; the second two ones to the bending energy of the fibres, again in $\alpha_{m}$-direction, having used the bending stiffness parameter $b_{i, j}^{\left(\alpha_{m}\right)}$; the last contribution is instead related to the shear energy of the springs with stiffness parameter $s_{i, j}^{(q)}$ where $q$ (ranging from 1 to 4) distinguishes each one of the four quadrants defined by the fibres around $P_{i, j}$-node forming the angle $\bar{\sigma}_{i, j}^{(q)}$ in the reference configuration.

Instead, the expression for the second gradient Piola's strain energy in the continuum model (in terms of the placement field $\chi$, see [2] for a depth insight, is given by:

$$
\begin{aligned}
W(\chi) & =\frac{1}{2} \int_{\Omega} \sum_{\alpha_{m}} A^{\left(\alpha_{m}\right)}\left(\left\|\mathbf{F} \mathbf{d}_{\alpha_{m}}\right\|-1\right)^{2} \mathrm{~d} \Omega \\
& +\frac{1}{2} \int_{\Omega} \sum_{\alpha_{m}} B^{\left(\alpha_{m}\right)}\left(\frac{\nabla \mathbf{F}\left|\mathbf{d}_{\alpha_{m}} \otimes \mathbf{d}_{\alpha_{m}} \cdot \nabla \mathbf{F}\right| \mathbf{d}_{\alpha_{m}} \otimes \mathbf{d}_{\alpha_{m}}}{\left\|\mathbf{F} \mathbf{d}_{\alpha_{m}}\right\|^{2}}-\left(\frac{\mathbf{F} \mathbf{d}_{\alpha_{m}}}{\left\|\mathbf{F} \mathbf{d}_{\alpha_{m}}\right\|} \cdot \frac{\nabla \mathbf{F} \mid \mathbf{d}_{\alpha_{m}} \otimes \mathbf{d}_{\alpha_{m}}}{\left\|\mathbf{F} \mathbf{d}_{\alpha_{m}}\right\|}\right)^{2}\right) \mathrm{d} \Omega \\
& +\frac{1}{2} \int_{\Omega} S\left(\arccos \left(\frac{\mathbf{F} \mathbf{d}_{\alpha_{1}}}{\left\|\mathbf{F} \mathbf{d}_{\alpha_{1}}\right\|} \cdot \frac{\mathbf{F} \mathbf{d}_{\alpha_{2}}}{\left\|\mathbf{F} \mathbf{d}_{\alpha_{2}}\right\|}\right)-\bar{\sigma}\right)^{2} \mathrm{~d} \Omega,
\end{aligned}
$$

where the stiffness parameters of the continuum model (macro) $A^{\left(\alpha_{m}\right)}, B^{\left(\alpha_{m}\right)}$ and $\mathrm{S}$ are related to the stiffness parameters of the discrete model (meso) $a_{i, j}^{\left(\alpha_{m}\right)}, b_{i, j}^{\left(\alpha_{m}\right)}$ and $s_{i, j}$ as follows:

$$
\begin{aligned}
A^{\left(\alpha_{m}\right)} & =\frac{a_{i, j}^{\left(\alpha_{m}\right)}}{\sin \bar{\sigma}}, \\
B^{\left(\alpha_{m}\right)} & =\frac{b_{i, j}^{\left(\alpha_{m}\right)}}{\sin \bar{\sigma}}, \\
S & =\frac{s_{i, j}}{\varepsilon^{2} \sin \bar{\sigma}},
\end{aligned}
$$

having used the notation

$$
\begin{aligned}
\mathbf{F} & =\nabla \chi, \\
\left(\nabla \mathbf{F} \mid \mathbf{d}_{\alpha_{m}} \otimes \mathbf{d}_{\alpha_{m}}\right)^{\beta} & =F_{\alpha_{m}, \alpha_{m}}^{\beta}, \quad\left(\text { no sum over repeated } \alpha_{m} \text { is intended }\right), \\
\sigma & =\arccos \left(\frac{\mathbf{F} \mathbf{d}_{\alpha_{1}}}{\left\|\mathbf{F} \mathbf{d}_{\alpha_{1}}\right\|} \cdot \frac{\mathbf{F} \mathbf{d}_{\alpha_{2}}}{\left\|\mathbf{F} \mathbf{d}_{\alpha_{2}}\right\|}\right)
\end{aligned}
$$

and considering that the area element $\mathrm{d} \Omega$ can be identified with $\varepsilon^{2} \sin \bar{\sigma}$ in the limit in which $\varepsilon$ approaches zero. 
Some remarks:

(1) In [2], Eqs. (2) and (3) were written in a more general form by using for the shear strain term the exponent $\gamma$ instead of 2 ; this is particularly useful to improve the fit of the experimental tests by theoretical models.

(2) The bending stiffness is expressed by means of the $\cos \beta_{i, j}^{\left(\alpha_{m}\right)}$ instead of the corresponding angle $\beta_{i, j}^{\left(\alpha_{m}\right)}$, these two possibilities are equivalent, in principle, but the first, avoiding the uses of $\arccos (\cdot)$ function, results more convenient from the computational point of view since it produces a more compact and effective code.

Having completely defined the strain energy of the pantographic structure, the solution consists in searching the complete equilibrium path, that is in a sequence of configurations defined by $p_{i, j}$, related to the non-dimensional parameter $u / L_{1}$, which satisfy the equilibrium equations deriving from the stationarity condition of the total potential energy. The reconstruction of the equilibrium path is pursued by means of an incremental-iterative procedure thoroughly described in [1].

\section{COMPARISON DISCRETE AND CONTINUUM MODEL WITH EXPERIMENTS}

The reader will remark that very few parameters are postulated to characterise both the discrete and the continuum models. On the contrary a wealth of experimental data are nearly perfectly fitted using these few parameters. In [2] an identification of the parameters of the continuum model in terms of the discrete model has been proposed. This strategy leads to the values reported in Table 1 for the springs stiffnesses, axial $a$, bending $b$ and shear $s$.

TABLE 1. Stiffnesses of axial $(a)$, bending $(b)$ and shear $(s)$ springs.

\begin{tabular}{cccc}
\hline$a(\mathrm{~N} / \mathrm{mm})$ & $b_{1}(\mathrm{Nmm})$ & $b_{2}(\mathrm{Nmm})$ & $s(\mathrm{Nmm})$ \\
\hline 165.6 & 148.9 & 148.9 & 0.977 \\
\hline
\end{tabular}

Using an in-house made code for discrete model, see [1,7, 8, 9] for details and results, and the Comsol Multiphysics for the second gradient continuum model, with the values springs stiffnesses reported in Table 1 we simulate the two traction tests, already discussed in Section 2, changing the orientation of the fibres.

In particular, to perform numerical simulations for the continuum model, a Hellinger-Reissner type variational principle is employed as done in $[2,11]$. In addition, cubic Hermite polinomial are used as shape functions in the FE scheme. The used meshes consist of 3250 quadrilateral elements for the case $\phi=120^{\circ}$ and 2800 quadrilateral elements for the case $\phi=60^{\circ}$ which correspond to 232388 degrees of freedom and 199658, respectively, see Fig. 10.

Figure 11 reports the strain energies and the global structural reaction when the non-dimensional assigned displacements $u / L_{1}$ on the right side increases $\left(u_{\max }=23.7 \mathrm{~mm}\right)$. In particular Fig. 11(a) reports the strain energies for the discrete (continuous line) and continuum (dot-dashed line) models divided into the three distinct components: axial, bending and shear energies.

Figure 11(b) reports the comparison between the global structural reaction $R$ (in $x_{1}$-direction) evaluated both by means of the discrete (red continuous line) and the continuum (red dot-dashed line). In addition, it is also reported the same quantity measured during the experimental test (black continuous line). We remark that both the closeness of the two curves obtained by means of the numerical simulations and the goodness of fitting the experimental evidence.

Figure 12 reports the density $r$ of the global structural reaction $R$ of the side $x_{1}=0$. This result is obtained by using the discrete model but, although non reported here, is confirmed by the 2 nd gradient continuum model. We highlight the negative (compression) values of $r_{1}$ in the central part of the side $x_{1}=0$ in a traction test. 
Somewhat interesting is the evolution of the deformation and of the strain energy. The discrete model furnishes the results reported in Fig. 13 for $u / u_{\max }=0.25,0.5,0.75$ and 1 (reference configuration is depicted in grey, colours show the strain energy density level).

Conversely, Fig. 14 reports the final deformation $\left(u=u_{\max }\right)$ along with the strain energy density level attained by using the second gradient continuum model.

Finally, for this test, Figure 15 reports an overlapping of the picture taken at the final stage of deformation $\left(u=u_{\max }\right)$ of the experiment and the correspondent deformation computed via numerical simulation using the discrete model: the overlapping clearly shows the capability of the numerical simulation to represent accurately the experiment.

The second test concern the problem depicted in Fig. 3. In this case the angle between non-parallel fibres is $\varphi=60^{\circ}$. Also in this case displacement-controlled traction test has been considered until a displacement $u_{1}=u_{\max }=74.7 \mathrm{~mm}$ on the side $x_{1}=L_{1}$.

Duplicating the same scheme of results of the first test, Figure 16 reports the strain energies and the global structural reaction when the non-dimensional assigned displacements $u / L_{1}$ on the right side increases $\left(u_{\max }=74.7 \mathrm{~mm}\right)$. In particular Fig. 16(a) reports the strain energies for the discrete (continuous line) and continuum (dot-dashed line) models divided into the three distinct components: axial, bending and shear energy.

Figure 16(b) reports the comparison between the global structural reaction $R$ evaluated both by means of the discrete (red continuous line) and the continuum (red dot-dashed line). In addition, it is also reported the same quantity measured during the experimental test (black continuous line). We remark that in this case there is a good agreement between the numerical simulations (that which uses the discrete model and that which considers the second gradient continuum model) but the experimental curve moves away from the simulations for value of the strain parameter $u / L_{1}$ greater than 0.2 .

Figure 17 reports the density $r$ of the global structural reaction $R$ of the side $x_{1}=0$. This result is obtained by using the discrete model but, although non reported here, is confirmed by the 2 nd gradient continuum model. We highlight the negative (compression) values of $r_{1}$ in the central part of the side $x_{1}=0$ in a traction test.

The deformation history computed by using the discrete model is reported in Fig. 18 for $u / u_{\max }=$ $0.25,0.5,0.75$ and 1 (reference configuration is depicted in grey, colours show the strain energy density level).

Figure 19 reports the final deformation $\left(u=u_{\max }\right)$ along with the strain energy density level computed by using the second gradient continuum model.

Also for this test a confirmation of the goodness of the results of numerical simulation is obtained overlapping the deformation of the experiment at the final stage with the correspondent deformation obtained via numerical simulation (the reported results are obtained by using the discrete model but those computed by using the second gradient continuum model are indistinguishable, see Figure 20.

We remark that the solid lines in the Figs. 14 and 19 are simply material lines in the actual configuration which correspond to the "real fibres" of the pantographic structure. Besides, the mesh adopted is chosen completely independent from the real arrangement of the fibres as shown in Fig. 10.

It is somewhat interesting to give some details about the times used to perform the numerical simulations advertising that the platforms are completely different. Indeed, the numerical simulations concerning the second gradient numerical model are obtained by using a $3 \mathrm{GHz}$ Intel core i7 equipped with 8 GB $1600 \mathrm{MHz}$ DDR3 RAM and the Comsol Multiphysics software: in this case we obtain the results for traction tests in 1555 seconds for the case of $\varphi=120^{\circ}$ and 1546 seconds for the case of $\varphi=60^{\circ}$. Conversely, the discrete model runs on the Matlab platform on a $3.5 \mathrm{GHz} 6$-Core Intel Xeon E5 equipped with 64 GB $1866 \mathrm{MHz}$ DDR3 RAM: in this case we obtain the results for traction test in 66 seconds for the case of $\varphi=120^{\circ}$ and 79 seconds for the case of $\varphi=60^{\circ}$. 


\section{CONCLUding REMARKS AND FUTURE PERSPECTIVES}

In $[5,4,12]$ more or less rigorous homogenization results are presented, in the framework of linear elasticity: i.e. small deformations and quadratic deformation energies. The model presented here, instead, tries to model the behavior of real pantographic structures undergoing large displacements. While the majority of the beams constituting the pantographic lattice are in the small deformation regime, we can however distinguish some boundary layers in which the involved beam elements are undergo very large deformations (more than 5\% of elongations, for instance, as remarked in [2]). These experimental evidence compelled us to introduce strongly nonlinear models in order to be able to design a priori pantographic sheet having tailored properties.

While the numerical simulations show a surprising agreement with experimental evidence, we feel that a rigorous basis on the homogenization results presented in [2] needs to be firmly established. We expect that $\Gamma$-convergence results can be now confidently formulated and conjectured, see [13]. Moreover we expect that the methods exploited in [14] could be adapted to get also a priori error estimates in the replacement process involved when passing from discrete to continuum models.

A further remark is needed: many cases of out of plane buckling of exotic pantographic sheets were observed. A phenomenological model proposed in $[15,16]$ has been successfully used to get qualitative predictions. However to get more general quantitative predictions an identification procedure involving discrete Lagrangian models with concentrated springs is needed, which applies to three-dimensional motion of two-dimensional pantographic sheets.

Finally, we list here some future developments and challenges:

(1) Although pantographic structures were conceived to give an example of second gradient metamaterial, see e.g. [17, 18, 19, 11, 20, 21, 22, 23, 24, 25, 26], the development of 3D printing technology allowed for the practical synthesis of such metamaterials. It deserves to be investigated how to improve the design of 3D printed fabrics in order to fully exploit the exotic behaviour of higher gradient metamaterials. We remark that, as seen in $[2,10]$, the behaviour of higher gradient continua shows many peculiarities which deserve deeper experimental investigations.

(2) The discrete nature of suitably designed beam lattices may be modelled also by means of more refined tools, see e.g. [27, 28, 29, 30, 31, 32, 33, 34] for an in-depth description of NURBS interpolation or using the generalized beam theory, see $[35,36]$, this in order to design even more complex metamaterials also in the 3D case where could be efficiently used the Pipkin model described in [37] and in the review paper [38].

(3) Another crucial point concerns the modelling of the breakdown evolution of pantographic sheets. Indeed some evidence has been already gathered about the onset and the evolution of failure. It is rather evident that have to be considered ruptures concerning both fibers and pivots. A first modelling effort to model such rupture phenomena was presented in [39] when the attention was limited to the rupture mechanism initiated by the rupture of a fiber, see also [40] for an insight on the modelling of fibres defects. In this context, surely deserve models able to consider the out-of-plane deformations and the related buckling phenomena, see [41, 42, 43, 44, 45] for a quick insight on this argument.

(4) The experimental identification of the parameters of the discrete model, i.e. the stiffnesses of the springs, require a specific investigation (see [46]). In particular methods of best fitting must be coupled to those used in extended sensitivity analysis by adapting, for example, the tools described in $[47,48]$ and exploited in $[49,50,51,52,53,54,48]$, see also [55, 56] for a more specific application to the description of huge and innovative structures.

(5) Experimental evidence shows the onset of some vibration phenomena in some specific experimental conditions. Therefore, it is relevant the extension of modelling to dynamic regimes, which can be obtained following the methods presented in see $[57,58]$ and also in $[59,60]$. The relevant analyses in nonlinear dynamic regimes could be performed also via semi-analyitical methods, such as the Multiple Scale Method, see [61, 62, 63, 64]. 
(6) The discrete Hencky-type model and the related numerical discretization technique could also be used to model granular media interactions, see [65], or generalized and micro-structured continua, see [17, 66, 67, 68, 69] and, in particular, [70, 71, 72, 73] for applications in civil engineering and [74] in biomechanics.

(7) In various experiments the contact between fibres was observed, if this kind of phenomenon has to be considered they could be interesting the guidelines reported in $[75,76]$.

\section{REFERENCES}

[1] E. Turco, F. dell'Isola, A. Cazzani, and N. L. Rizzi. Hencky-type discrete model for pantographic structures: numerical comparison with second gradient continuum models. Zeitschrift für Angewandte Mathematik und Physik, 67(4):1-28, August 2016.

[2] F. dell'Isola, I. Giorgio, M. Pawlikowski, and N. L. Rizzi. Large deformations of planar extensible beams and pantographic lattices: Heuristic homogenisation, experimental and numerical examples of equilibrium. Proceedings of the Royal Society of London A: Mathematical, Physical and Engineering Sciences, 472(2185), 2016.

[3] F. dell'Isola, T. Lekszycki, M. Pawlikowski, R. Grygoruk, and L. Greco. Designing a light fabric metamaterial being highly macroscopically tough under directional extension: first experimental evidence. Zeitschrift für angewandte Mathematik und Physik, 66(6):3473-3498, 2015.

[4] J.-J. Alibert, P. Seppecher, and F. dell'Isola. Truss modular beams with deformation energy depending on higher displacement gradients. Mathematics and Mechanics of Solids, 8(1):51-73, 2003.

[5] J.-J. Alibert and A. Della Corte. Second-gradient continua as homogenized limit of pantographic microstructured plates: a rigorous proof. Zeitschrift für Angewandte Mathematik und Physik, 66(5):2855-2870, 2015.

[6] A. Madeo, A. Della Corte, L. Greco, and P. Neff. Wave propagation in pantographic 2D lattices with internal discontinuities. Proceedings of the Estonian Academy of Sciences, 64(3S):325-330, 2015.

[7] E. Turco, M. Golaszewski, A. Cazzani, and N. L. Rizzi. Large deformations induced in planar pantographic sheets by loads applied on fibers: experimental validation of a discrete Lagrangian model. Mechanics Research Communications, 76:51-56, 2016.

[8] E. Turco, K. Barcz, M. Pawlikowski, and N. L. Rizzi. Non-standard coupled extensional and bending bias tests for planar pantographic lattices. Part I: numerical simulations. Zeitschrift für Angewandte Mathematik und Physik, 67(122):1-16, 2016.

[9] E. Turco, K. Barcz, and N. L. Rizzi. Non-standard coupled extensional and bending bias tests for planar pantographic lattices. Part II: comparison with experimental evidence. Zeitschrift für Angewandte Mathematik und Physik, 67(123):1-16, 2016.

[10] F. dell'Isola, U. Andreaus, and L. Placidi. At the origins and in the vanguard of peridynamics, nonlocal and higher-gradient continuum mechanics: An underestimated and still topical contribution of Gabrio Piola. Mathematics and Mechanics of Solids, 20(8):887-928, 2015.

[11] D. Scerrato, I. A. Zhurba Eremeeva, T. Lekszycki, and N. L. Rizzi. On the effect of shear stiffness on the plane deformation of linear second gradient pantographic sheets. Zeitschrift für Angewandte Mathematik und Mechanik, 96(11):1268-1279, 2016.

[12] C. Boutin, F. dell'Isola, I. Giorgio, and L. Placidi. Linear pantographic sheets. Part I: Asymptotic micro-macro models identification. submitted, 2016.

[13] A. Braides, A. Causin, and M. Solci. Interfacial energies on quasicrystals. IMA Journal of Applied Mathematics, 77(6):816-836, 2012.

[14] A. Carcaterra, F. dell'Isola, R. Esposito, and M. Pulvirenti. Macroscopic description of microscopically strongly inhomogenous systems: A mathematical basis for the synthesis of higher gradients metamaterials. Archive for Rational Mechanics and Analysis, (doi:10.1007/s00205- 015-0879-5), 2015. 
[15] D. J. Steigmann and F. dell'Isola. Mechanical response of fabric sheets to three-dimensional bending, twisting, and stretching. Acta Mechanica Sinica, 31(3):373-382, 2015.

[16] D. Scerrato, I. Giorgio, and N. L. Rizzi. Three-dimensional instabilities of pantographic sheets with parabolic lattices: numerical investigations. Zeitschrift für Angewandte Mathematik und Physik, 67(3):1-19, 2016.

[17] F. dell'Isola, D. Steigmann, and A. Della Corte. Synthesis of fibrous complex structures: Designing microstructure to deliver targeted macroscale response. Applied Mechanics Reviews, 67(6):060804, 2015.

[18] I. Giorgio, R. Grygoruk, F. dell'Isola, and D. J. Steigmann. Pattern formation in the threedimensional deformations of fibered sheets. Mechanics Research Communications, 69:164-171, 2015.

[19] D. Scerrato, I. Giorgio, A. Della Corte, A. Madeo, and A. Limam. A micro-structural model for dissipation phenomena in the concrete. International Journal for Numerical and Analytical Methods in Geomechanics, 39(18):2037-2052, 2015.

[20] M. V. D’Agostino, I. Giorgio, L. Greco, A. Madeo, and P. Boisse. Continuum and discrete models for structures including (quasi-) inextensible elasticae with a view to the design and modeling of composite reinforcements. International Journal of Solids and Structures, 59:1-17, 2015.

[21] L. Placidi, U. Andreaus, and I. Giorgio. Identification of two-dimensional pantographic structure via a linear d4 orthotropic second gradient elastic model. Journal of Engineering Mathematics, doi: 10.1007/s10665- 016-9856-8, 2016.

[22] L. Placidi and A. E. Dhaba. Semi-inverse method à la Saint-Venant for two-dimensional linear isotropic homogeneous second-gradient elasticity. Mathematics and Mechanics of Solids, doi: 10.1177/1081286515616043, 2015.

[23] F. dell'Isola, I. Giorgio, and U. Andreaus. Elastic pantographic 2d lattices: A numerical analysis on static response and wave propagation. Proceedings of the Estonian Academy of Sciences, 64(3):219225, 2015.

[24] F. dell'Isola, A. Della Corte, L. Greco, and A. Luongo. Plane bias extension test for a continuum with two inextensible families of fibers: A variational treatment with lagrange multipliers and a perturbation solution. International Journal of Solids and Structures, 81:1-12, 2016.

[25] M. Cuomo, F. dell' Isola, and L. Greco. Simplified analysis of a generalized bias test for fabrics with two families of inextensible fibres. Zeitschrift für angewandte Mathematik und Physik, 67(3):1-23, 2016.

[26] F. dell'Isola, M. Cuomo, L. Greco, and A. Della Corte. Bias extension test for pantographic sheets: numerical simulations based on second gradient shear energies. Journal of Engineering Mathematics, doi:10.1007/s10665-016-9865-7, 2016.

[27] A. Cazzani, M. Malagù, and E. Turco. Isogeometric analysis of plane curved beams. Mathematics and Mechanics of Solids, 21(5):562-577, 2016.

[28] A. Cazzani, M. Malagù, and E. Turco. Isogeometric analysis: a powerful numerical tool for the elastic analysis of historical masonry arches. Continuum Mechanics and Thermodynamics, 28(1):139156, 2016.

[29] A. Cazzani, M. Malagù, E. Turco, and F. Stochino. Constitutive models for strongly curved beams in the frame of isogeometric analysis. Mathematics and Mechanics of Solids, 21(2):182-209, 2016.

[30] A. Bilotta, G. Formica, and E. Turco. Performance of a high-continuity finite element in threedimensional elasticity. International Journal for Numerical Methods in Biomedical Engineering, 26:1155-1175, 2010.

[31] L. Greco and M. Cuomo. B-Spline interpolation of Kirchhoff-Love space rods. Computer Methods in Applied Mechanics and Engineering, 256:251-269, 2013.

[32] L. Greco and M. Cuomo. An implicit $G^{1}$ multi patch B-spline interpolation for Kirchhoff-Love space rod. Computer Methods in Applied Mechanics and Engineering, 269:173-197, 2014. 
[33] L. Greco and M. Cuomo. An isogeometric implicit G1 mixed finite element for Kirchhoff space rods. Computer Methods in Applied Mechanics and Engineering, 298:325-349, 2016.

[34] A. Cazzani, F. Stochino, and E. Turco. An analytical assessment of finite elements and isogeometric analysis of the whole spectrum of Timoshenko beams. Zeitschrift für Angewandte Mathematik und Mechanik, 96(10):1220-1244, 2016.

[35] G. Piccardo, G. Ranzi, and A. Luongo. A complete dynamic approach to the generalized beam theory cross-section analysis including extension and shear modes. Mathematics and Mechanics of Solids, 19(8):900-924, 2014.

[36] G. Piccardo, G. Ranzi, and A. Luongo. A direct approach for the evaluation of the conventional modes within the gbt formulation. Thin-Walled Structures, 74:133-145, 2014.

[37] L. Placidi, L. Greco, S. Bucci, E. Turco, and N. L. Rizzi. A second gradient formulation for a 2D fabric sheet with inextensible fibres. Zeitschrift für angewandte Mathematik und Physik, 67(114):124, 2016.

[38] L. Placidi, E. Barchiesi, E. Turco, and N. L. Rizzi. A review on 2D models for the description of pantographic fabrics. Zeitschrift für angewandte Mathematik und Physik, 67(121):1-20, 2016.

[39] E. Turco, F. dell'Isola, N. L. Rizzi, R. Grygoruk, W. H. Müller, and C. Liebold. Fiber rupture in sheared planar pantographic sheets: numerical and experimental evidence. Mechanics Research Communications, 76:86-90, 2016.

[40] E. Turco and N. L. Rizzi. Pantographic structures presenting statistically distributed defects: numerical investigations of the effects on deformation fields. Mechanics Research Communications, 77:65-69, 2016.

[41] F. D’Annibale, G. Rosi, and A. Luongo. Linear stability of piezoelectric-controlled discrete mechanical systems under nonconservative positional forces. Meccanica, 50(3):825-839, 2015.

[42] N. Rizzi, V. Varano, and S. Gabriele. Initial postbuckling behavior of thin-walled frames under mode interaction. Thin-Walled Structures, 68:124-134, 2013.

[43] S. Gabriele, N. Rizzi, and V. Varano. A 1D higher gradient model derived from Koiter's shell theory. Mathematics and Mechanics of Solids, 21(6):737-746, 2016.

[44] H. AminPour and N. Rizzi. A one-dimensional continuum with microstructure for single-wall carbon nanotubes bifurcation analysis. Mathematics and Mechanics of Solids, 21(2):168-181, 2016.

[45] S. Gabriele, N. L. Rizzi, and V. Varano. A 1D nonlinear TWB model accounting for in plane cross-section deformation. International Journal of Solids and Structures, 94-95:170-178, 2016.

[46] L. Placidi, U. Andreaus, A. Della Corte, and T. Lekszycki. Gedanken experiments for the determination of two-dimensional linear second gradient elasticity coefficients. Zeitschrift für Angewandte Mathematik und Physik (ZAMP), 66(6):3699-3725, 2015.

[47] E. Turco. Tools for the numerical solution of inverse problems in structural mechanics: review and research perspectives. European Journal of Environmental and Civil Engineering, doi: 10.1080/19648189.2015.1134673:1-46, 2016.

[48] E. Turco. Identification of axial forces on statically indeterminate pin-jointed trusses by a nondestructive mechanical test. The Open Civil Engineering Journal, 7:50-57, 2013.

[49] T. Lekszycki, N. Olhoff, and J. J. Pedersen. Modelling and identification of viscoelastic properties of vibrating sandwich beams. Composite Structures, 22(1):15-31, 1992.

[50] A. Bilotta and E. Turco. A numerical study on the solution of the Cauchy problem in elasticity. International Journal of Solids and Structures, 46:4451-4477, 2009.

[51] A. Bilotta, A. Morassi, and E. Turco. Reconstructing blockages in a symmetric duct via quasiisospectral horn operators. Journal of Sound and Vibration, 366:149-172, 2016.

[52] A. Bilotta and E. Turco. Numerical sensitivity analysis of corrosion detection. Mathematics and Mechanics of Solids, doi: 10.1177/1081286514560093:1-17, 2014.

[53] G. Alessandrini, A. Bilotta, G. Formica, A. Morassi, E. Rosset, and E. Turco. Evaluating the volume of a hidden inclusion in an elastic body. Journal of Computational and Applied Mathematics, 198(2):288-306, 2007. 
[54] G. Alessandrini, A. Bilotta, A. Morassi, and E. Turco. Computing volume bounds of inclusions by EIT measurements. Journal of Scientific Computing, 33(3):293-312, 2007.

[55] F. Buffa, A. Cazzani, A. Causin, S. Poppi, G. M. Sanna, M. Solci, F. Stochino, and E. Turco. The Sardinia Radio Telescope: a comparison between close range photogrammetry and FE models. Mathematics and Mechanics of Solids, doi: 10.1177/1081286515616227:1-22, 2015.

[56] F. Stochino, A. Cazzani, S. Poppi, and E. Turco. Sardinia Radio Telescope finite element model updating by means of photogrammetric measurements. Mathematics and Mechanics of Solids, doi: 10.1177/1081286515616046:1-17, 2015.

[57] D. Del Vescovo and I. Giorgio. Dynamic problems for metamaterials: review of existing models and ideas for further research. International Journal of Engineering Science, 80:153-172, 2014.

[58] A. Battista, C. Cardillo, D. Del Vescovo, N. L. Rizzi, and E. Turco. Frequency shifts induced by large deformations in planar pantographic continua. Nanomechanics Science and Technology: An International Journal, 6(2):161-178, 2015.

[59] A. Cazzani, F. Stochino, and E. Turco. On the whole spectrum of Timoshenko beams. Part I: a theoretical revisitation. Zeitschrift für Angewandte Mathematik und Physik, 67(24):1-30, 2016.

[60] A. Cazzani, F. Stochino, and E. Turco. On the whole spectrum of Timoshenko beams. Part II: further applications. Zeitschrift für Angewandte Mathematik und Physik, 67(25):1-21, 2016.

[61] A. Luongo and A. Paolone. Perturbation methods for bifurcation analysis from multiple nonresonant complex eigenvalues. Nonlinear Dynamics, 14(3):193-210, 1997.

[62] A. Luongo and A. Paolone. Multiple scale analysis for divergence-Hopf bifurcation of imperfect symmetric systems. Journal of Sound and Vibration, 218(3):527-539, 1998.

[63] A. Luongo and F. D'Annibale. Double zero bifurcation of nonlinear viscoelastic beams under conservative and non conservative loads. International Journal of Non-Linear Mechanics, 55:128139, 2013.

[64] A. Luongo, F. D'Annibale, and M. Ferretti. Hard loss of stability of Ziegler's column with nonlinear damping. Meccanica, 51(11):2647-2663, 2016.

[65] A. Misra and P. Poorsolhjouy. Granular micromechanics based micromorphic model predicts frequency band gaps. Continuum Mechanics and Thermodynamics, 28(1):215-234, 2016.

[66] J. Altenbach, H. Altenbach, and V. A. Eremeyev. On generalized Cosserat-type theories of plates and shells: a short review and bibliography. Archive of Applied Mechanics, 80(1):73-92, 2010.

[67] V. A. Eremeyev and W. Pietraszkiewicz. Material symmetry group and constitutive equations of micropolar anisotropic elastic solids. Mathematics and Mechanics of Solids, (doi: 10.1177/1081286515582862), 2015.

[68] F. Dos Reis and J. F. Ganghoffer. Construction of micropolar continua from the asymptotic homogenization of beam lattices. Computers $\mathcal{E}$ Structures, 112-113:354-363, 2012.

[69] K. Elnady, F. Dos Reis, and J.-F. Ganghoffer. Construction of second order gradient continuous media by the discrete asymptotic homogenization method. International Journal of Applied Mechanics, 2014.

[70] C. Caggegi, V. Pensée, M. Fagone, M. Cuomo, and L. Chevalier. Experimental global analysis of the efficiency of carbon fiber anchors applied over cfrp strengthened bricks. Construction and Building Materials, 53:203-212, 2014.

[71] F. Tedesco, A. Bilotta, and E. Turco. Multiscale 3D mixed FEM analysis of historical masonry constructions. European Journal of Environmental and Civil Engineering, doi: 10.1080/19648189.2015.1134676, 2016.

[72] A. Bilotta and E. Turco. Equilibrium path for elastoplastic pressure-sensitive materials via an effective 3D mixed finite element. ZAMM - Zeitschrift für Angewandte Mathematik und Mechanik, doi: 10.1002/zamm.201600051:1-15, 2016.

[73] T. Lekszycki, S. Bucci, D. Del Vescovo, E. Turco, and N. L. Rizzi. A comparison between different approaches for modelling media with viscoelastic properties via optimization analyses. 
ZAMM - Zeitschrift für Angewandte Mathematik und Mechanik, DOI 10.1002/zamm.201600121:117, 2016.

[74] A. Tomic, A. Grillo, and S. Federico. Poroelastic materials reinforced by statistically oriented fibres - numerical implementation and application to articular cartilage. IMA Journal of Applied Mathematics, 79:1027-1059, 2014.

[75] U. Andreaus, B. Chiaia, and L. Placidi. Soft-impact dynamics of deformable bodies. Continuum Mechanics and Thermodynamics, 25:375-398, 2013.

[76] U. Andreaus, P. Baragatti, and L. Placidi. Experimental and numerical investigations of the responses of a cantilever beam possibly contacting a deformable and dissipative obstacle under harmonic excitation. International Journal of Non-Linear Mechanics, 80:96-106, 2016.

Department of Architecture, Design and Urban Planning, University of Sassari, Italy

E-mail address: emilio.turco@uniss.it

DCEBM, Warsaw University of Technology, Poland

E-mail address: golaszewski .maciej07@gmail.com

Faculty of Engineering, University of Roma "La Sapienza”, Roma, Italy

E-mail address: ivan.giorgio@uniroma1.it

Faculty of Engineering, DiCEAA, University of L'Aquila, L'Aquila, Italy

E-mail address: francesco.dannibale@univaq.it 


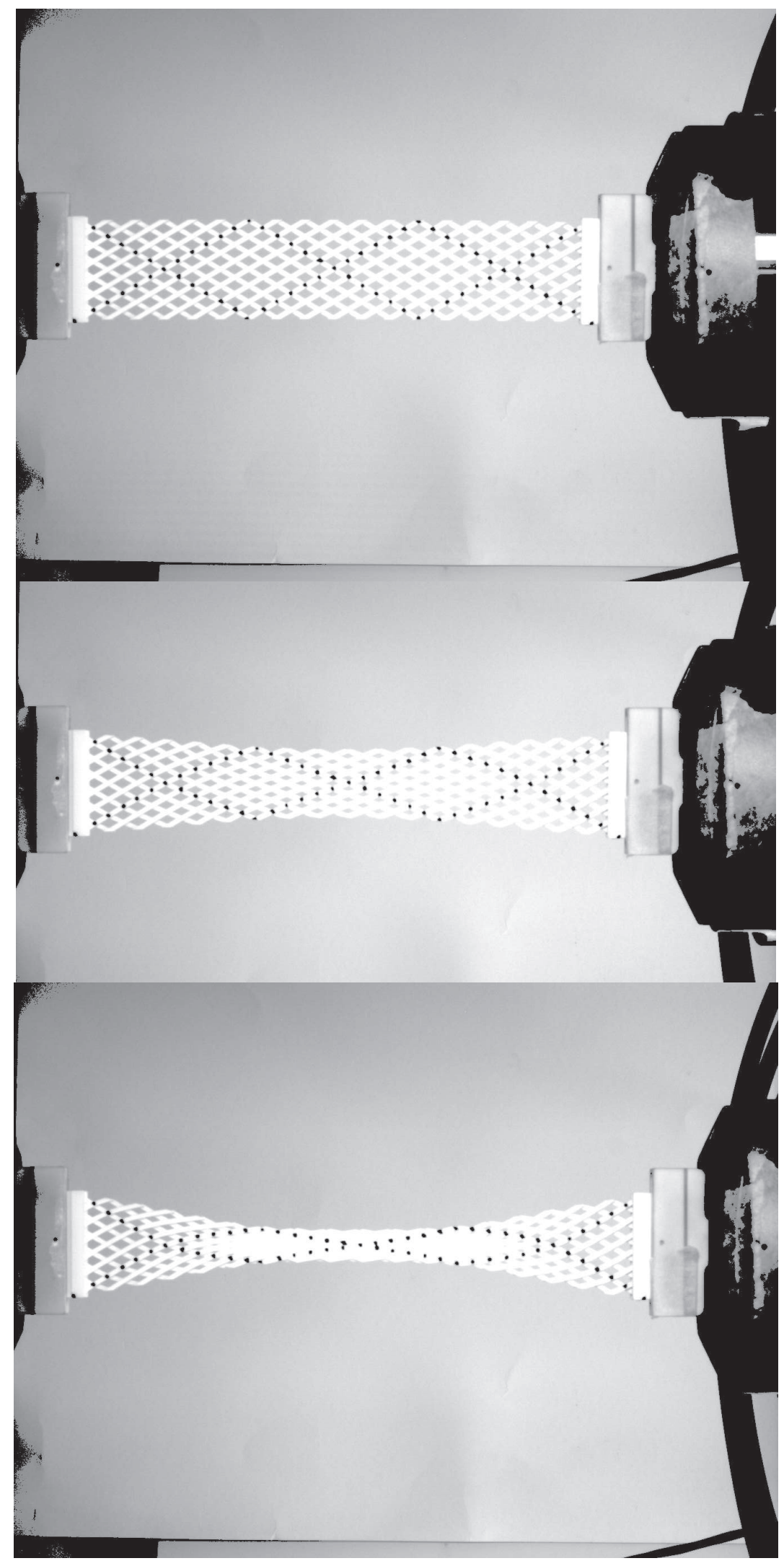

FIGURE 4. Pantographic lattice with non-orthogonal fibers $\left(\varphi=120^{\circ}\right)$ : sequence of deformations. 


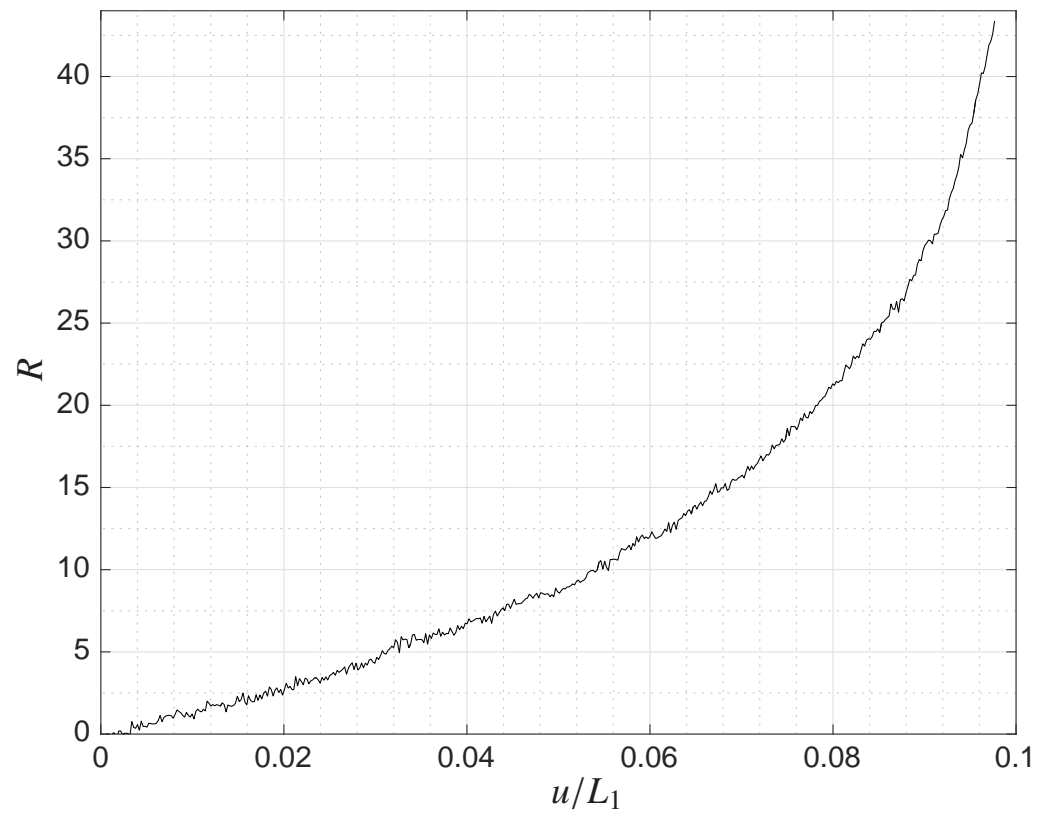

Figure 5. Pantographic lattice with non-orthogonal fibers $\left(\varphi=120^{\circ}\right)$ : global structural reaction $R$ vs. $u / L_{1}$. 


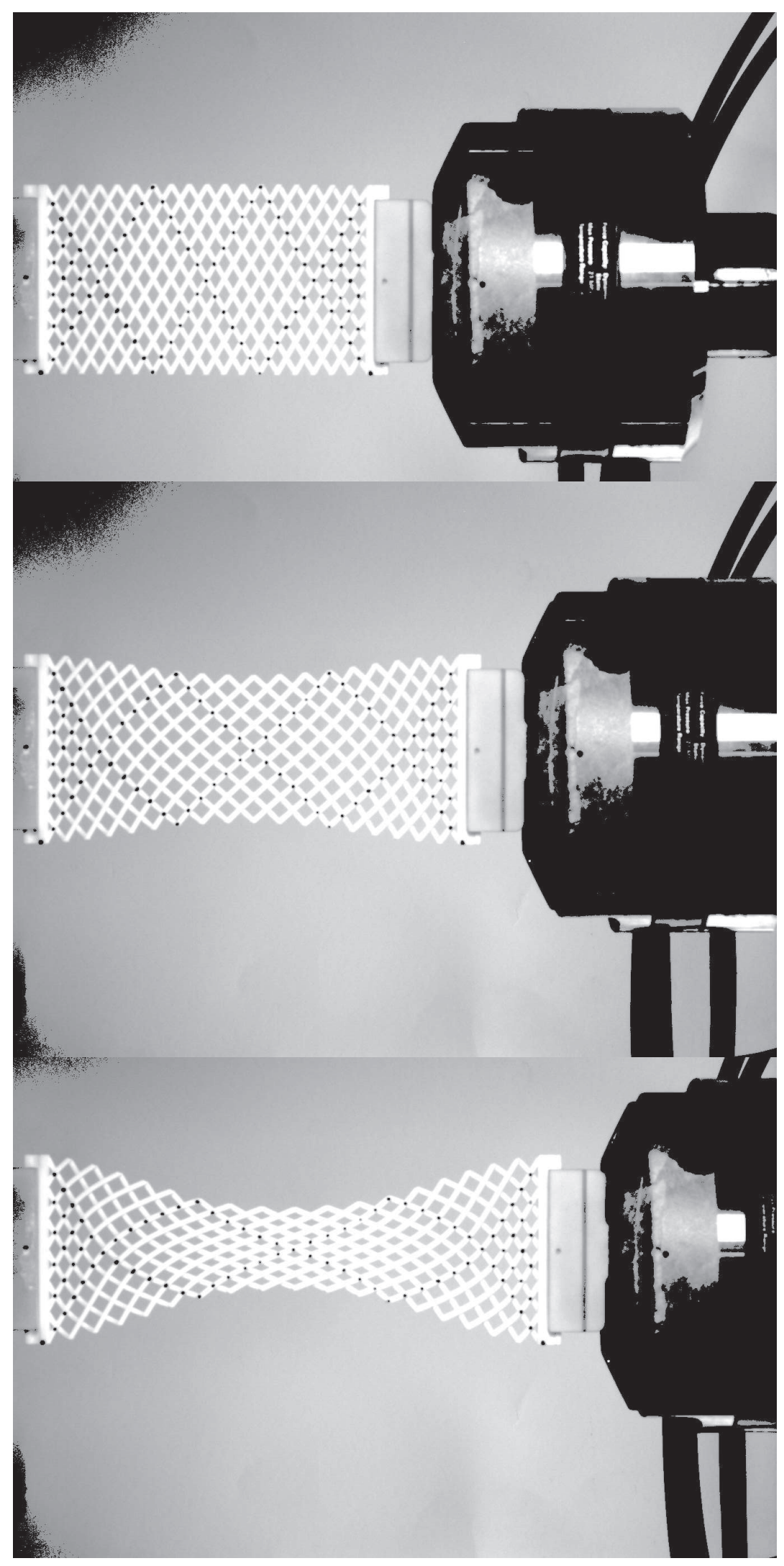

Figure 6. Pantographic lattice with non-orthogonal fibers $\left(\varphi=60^{\circ}\right)$ : sequence of deformations. 


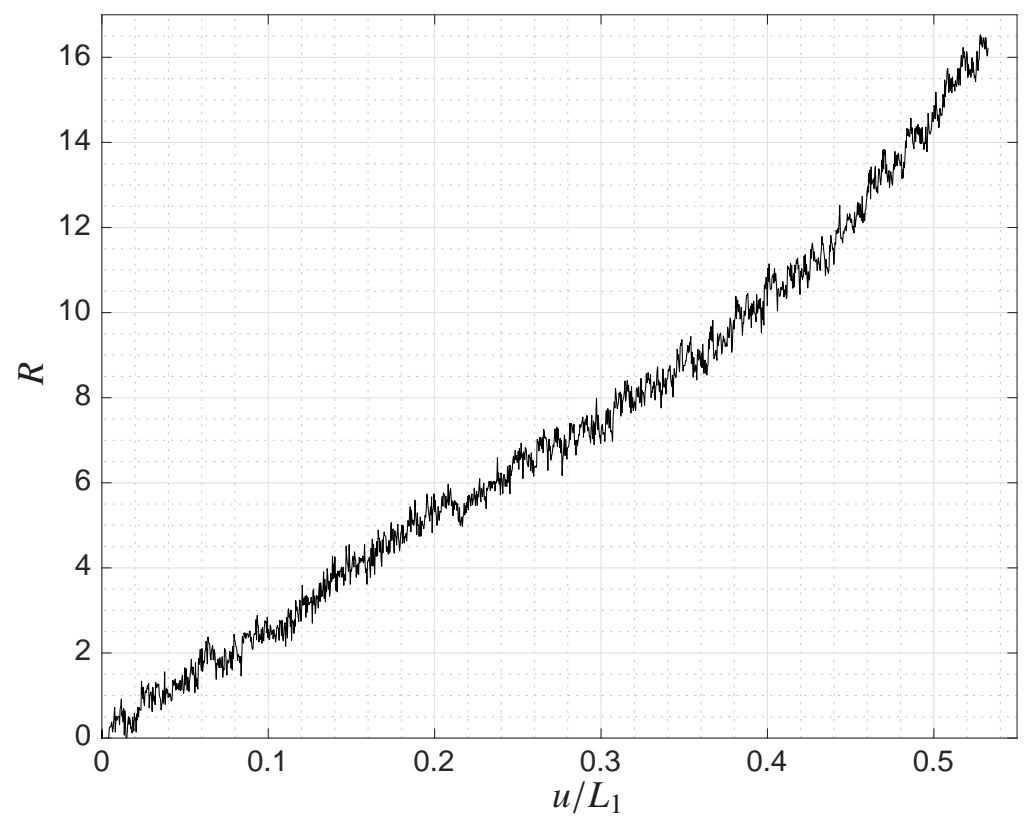

Figure 7. Pantographic lattice with non-orthogonal fibres $\left(\varphi=60^{\circ}\right)$ : global structural reaction $R$ vs. $u / L_{1}$.

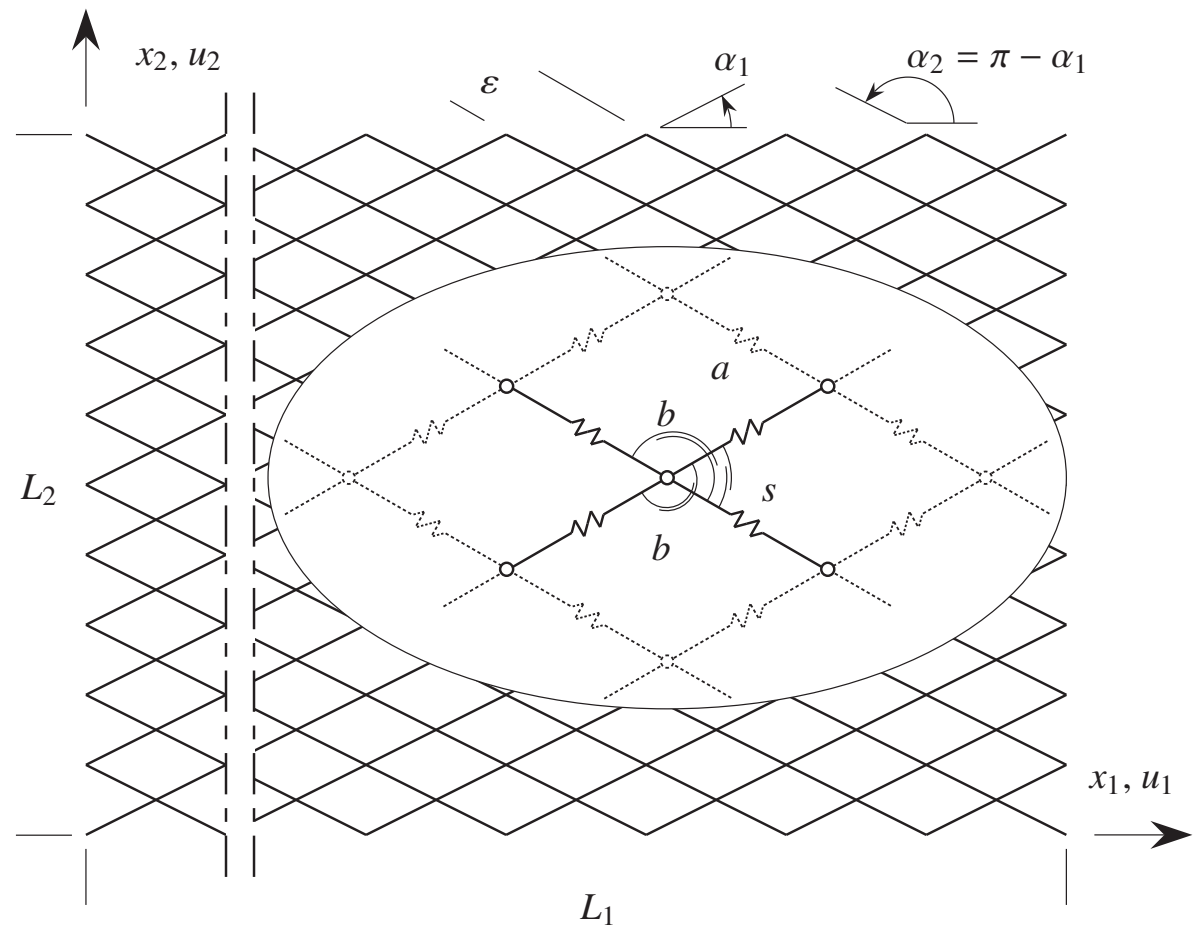

Figure 8. Hencky-type mechanical model of a pantographic lattice. 


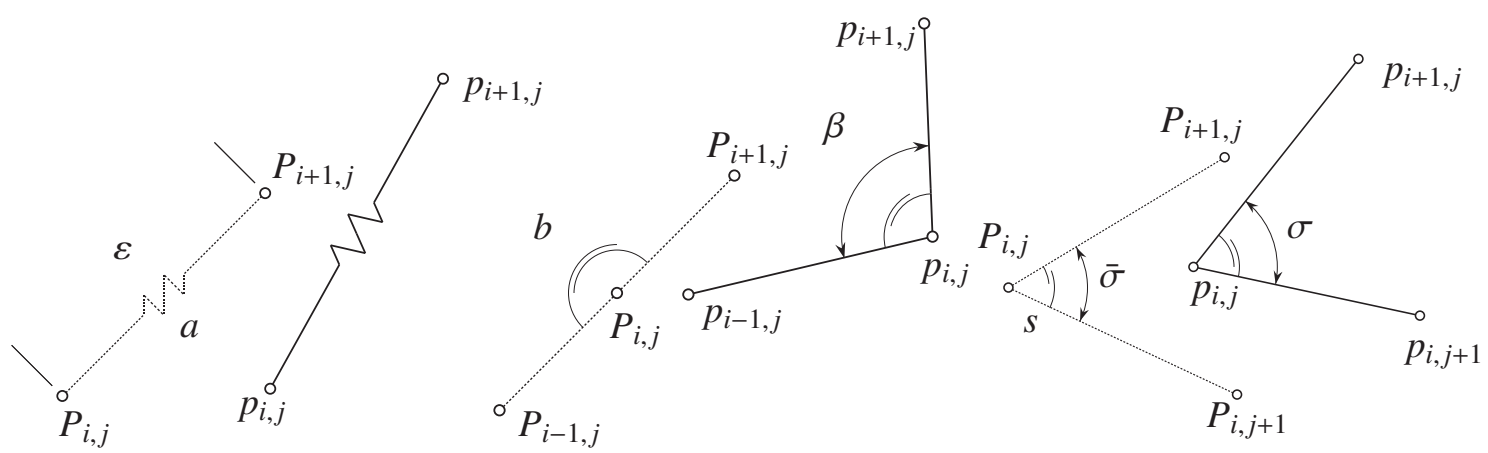

(a) axial spring

(b) bending spring

(c) shear spring

Figure 9. Kinematics of axial (a), bending (b) and shear (c) springs: reference (dashed line) and current (continuous line) configurations.

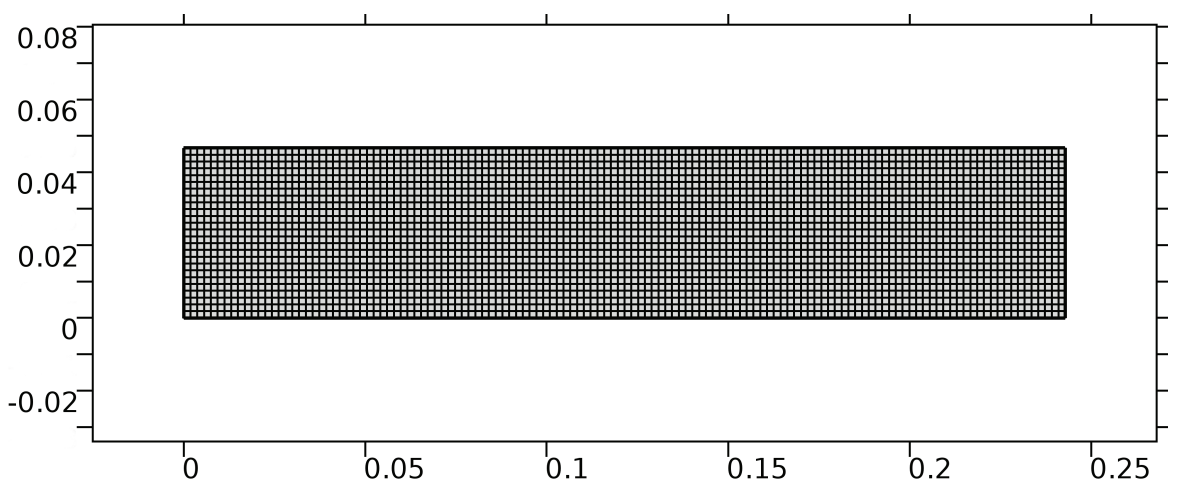

(a) $\varphi=120^{\circ}$

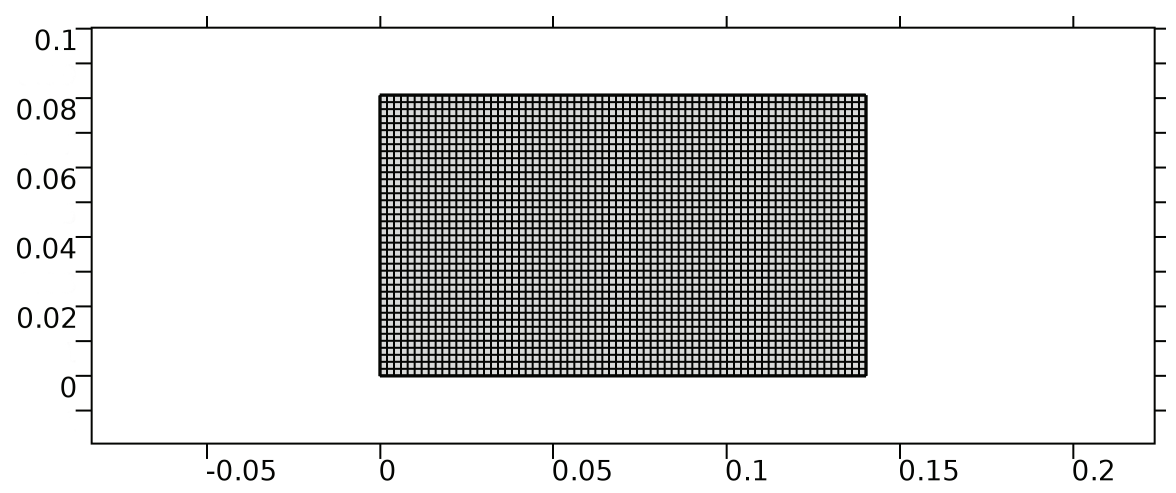

(b) $\varphi=60^{\circ}$

FIGURE 10. Meshes used for the simulation of the traction tests by using the second gradient numerical model: $\varphi=120^{\circ}$ (a) and $\varphi=60^{\circ}(\mathrm{b}$ ). 


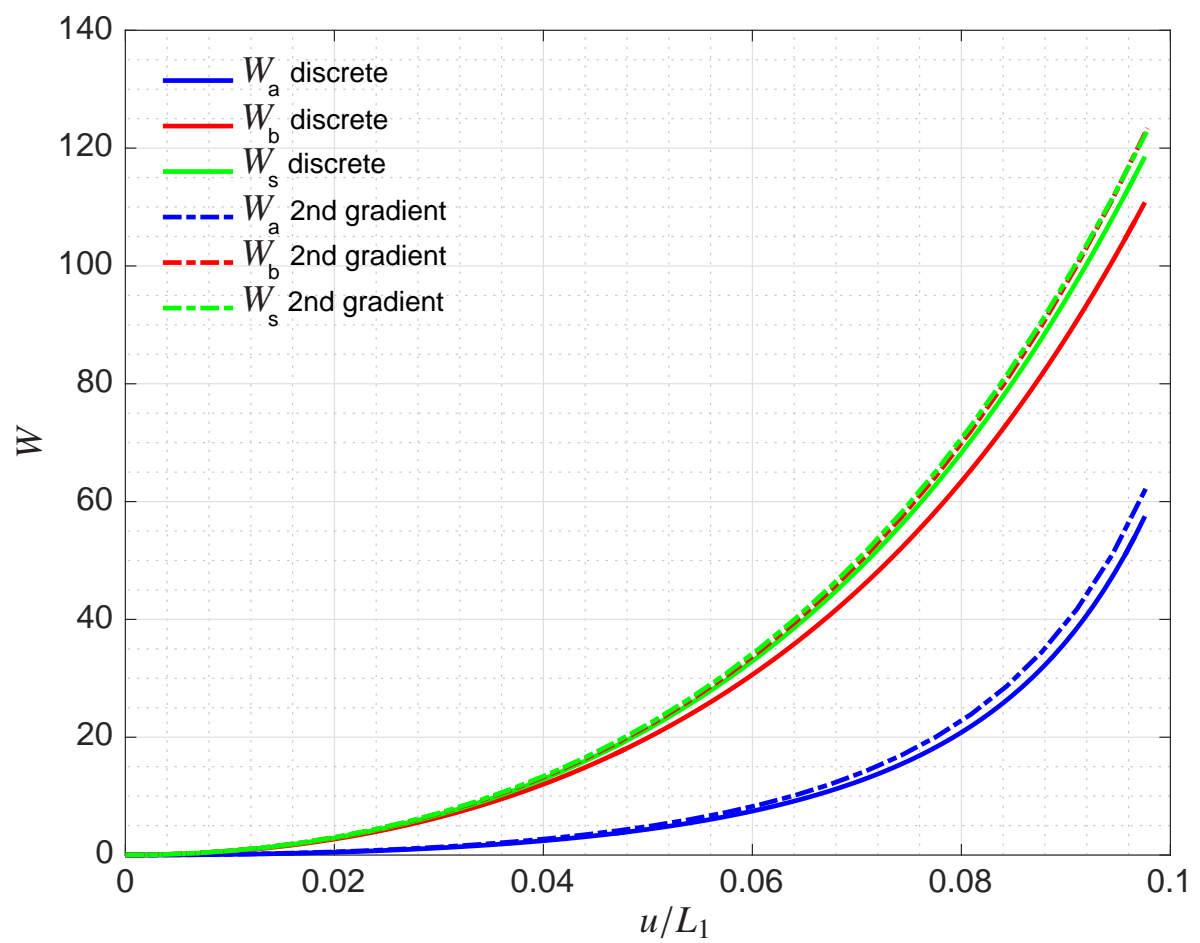

(a) Strain energy $W$ vs. $u / L_{1}$ distinct for axial (blue), bending (red) and shear (green) contribution for the discrete (continuous line) and 2nd gradient continuum (dot-dashed) model.

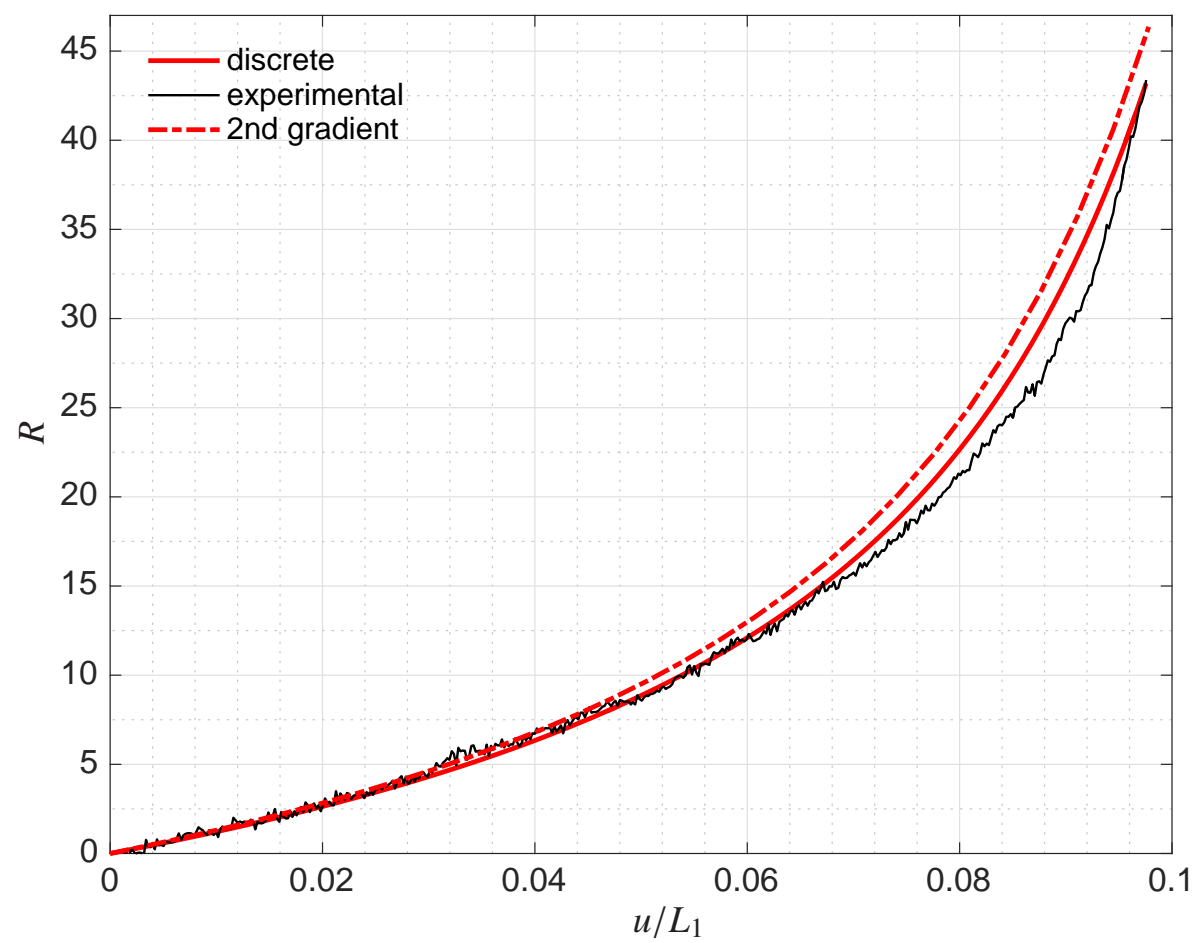

(b) Global structural reaction $R$ vs. $u / L_{1}$ for the discrete model (continuous red line), 2nd gradient continuum model (dot-dashed red line) and for the experiment (continuous black line).

Figure 11. Pantographic lattice with non-orthogonal fibres $\left(\varphi=120^{\circ}\right)$ : strain energy $W$ and global structural reaction $R$ for discrete and 2nd gradient continuum model. 


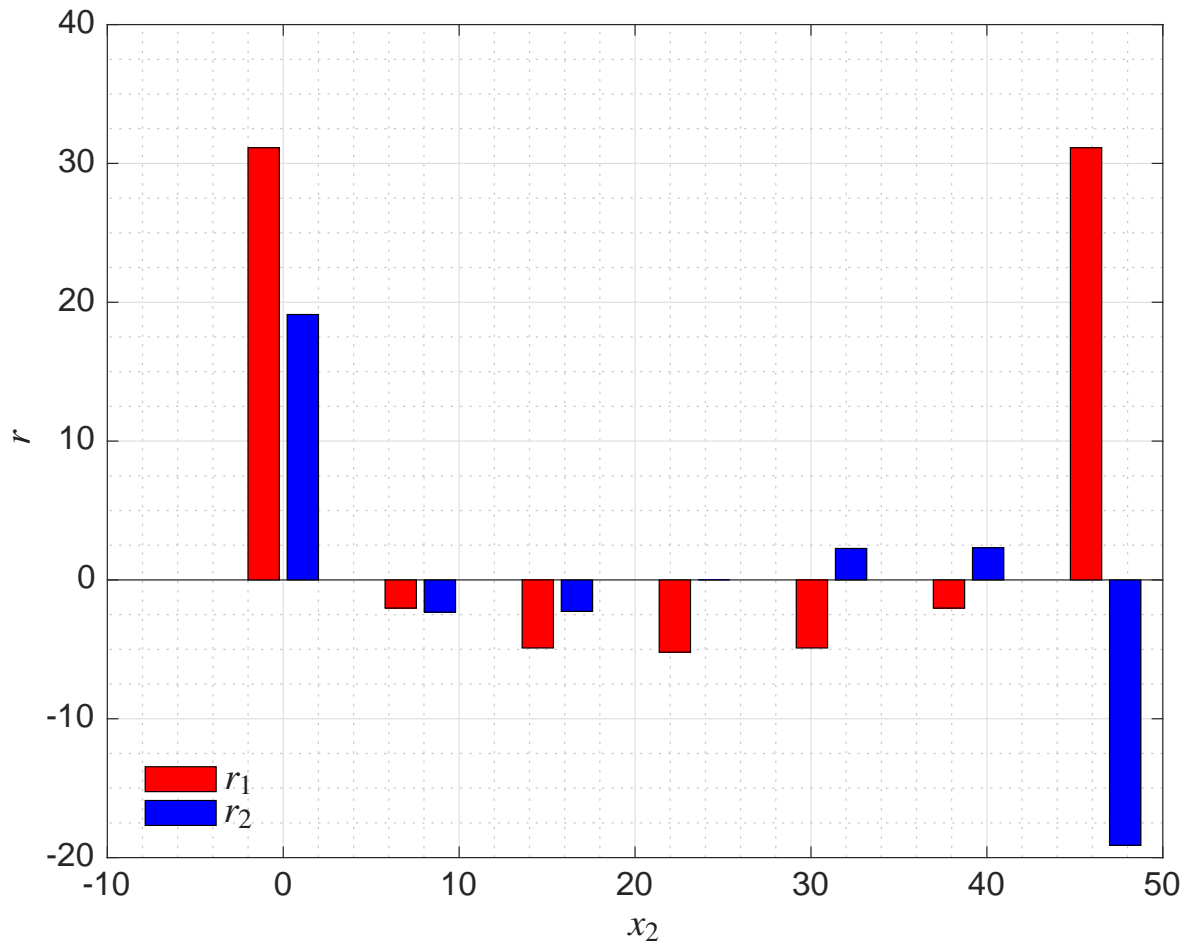

FIGURE 12. Pantographic lattice with non-orthogonal fibres $\left(\varphi=120^{\circ}\right)$ : density of global structural reaction $r$ on the side $x_{1}=0$ for $u=u_{\max }$. 

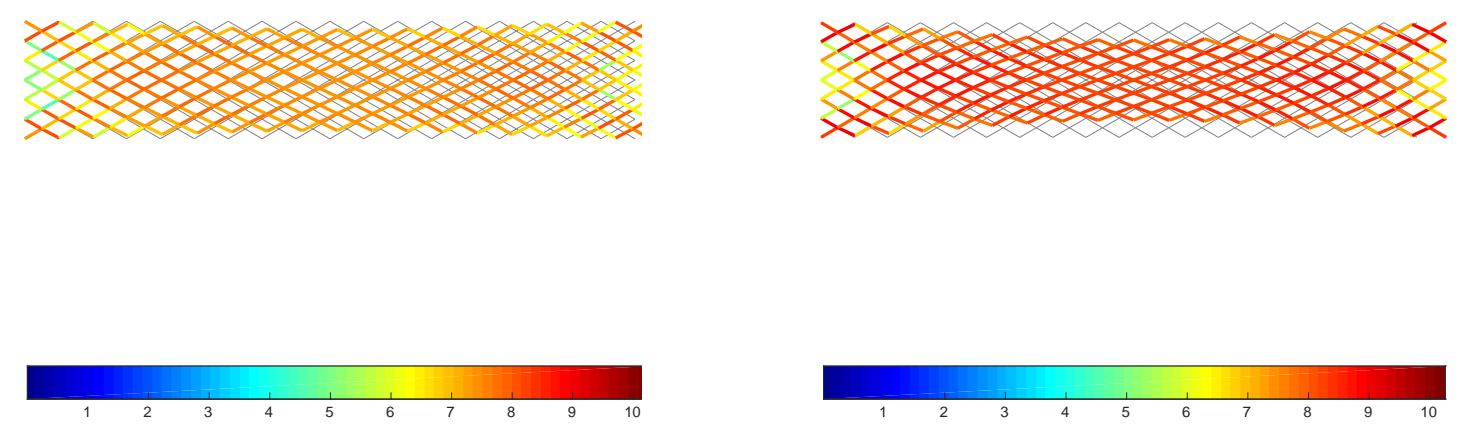

(a) $u / u_{\max }=0.25$

(b) $u / u_{\max }=0.5$
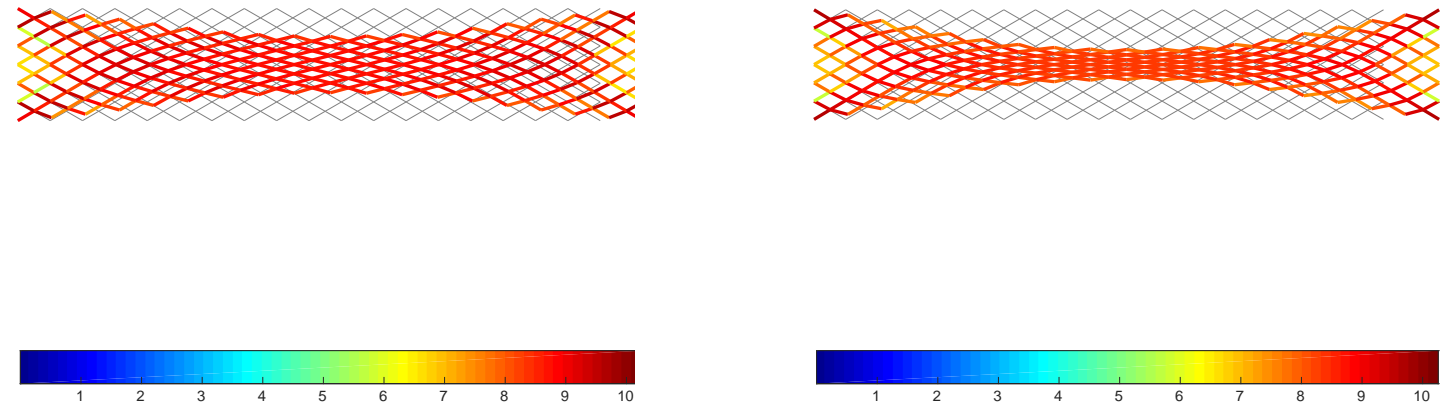

(c) $u / u_{\max }=0.75$

(d) $u / u_{\max }=1$

Figure 13. Pantographic lattice with non-orthogonal fibres $\left(\varphi=120^{\circ}\right)$ : history of deformation by using the Hencky-type numerical model (reference configuration is depicted in grey, colours indicate the strain energy level).

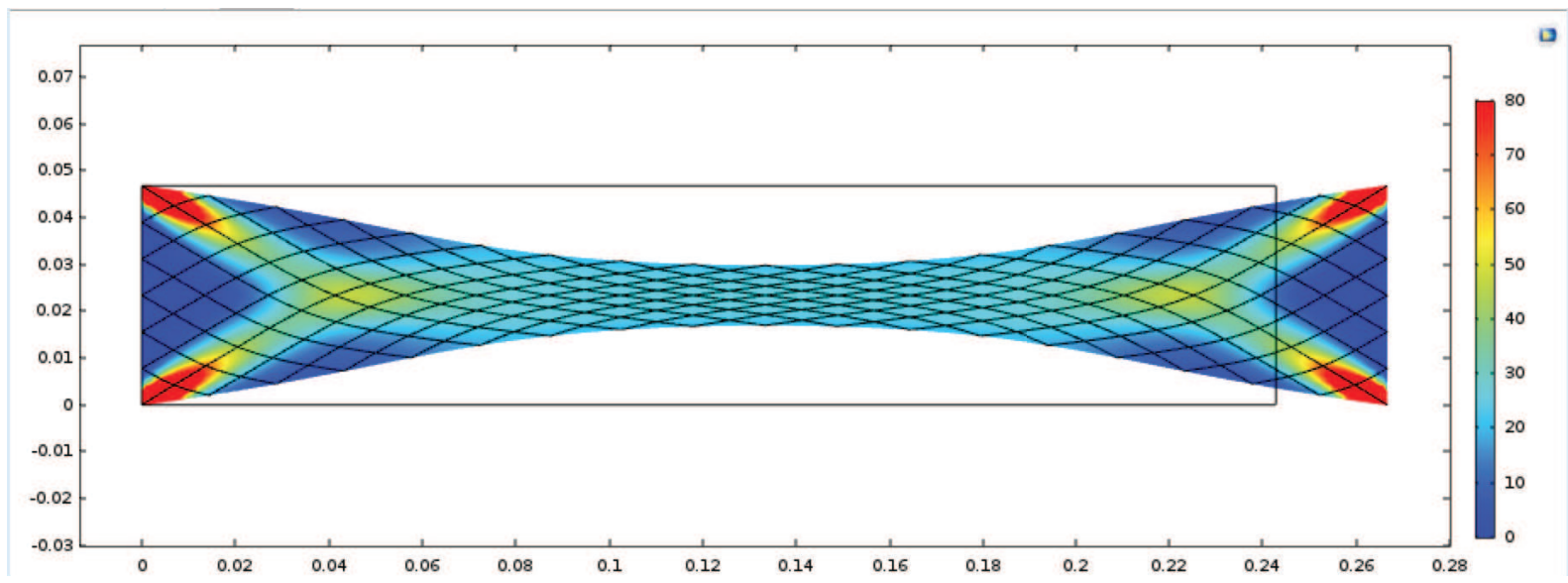

FIgURE 14. Pantograph lattice with non-orthogonal fibres $\left(\varphi=120^{\circ}\right)$ : deformation computed by the 2 nd gradient continuum model (colours represent the energy level). 


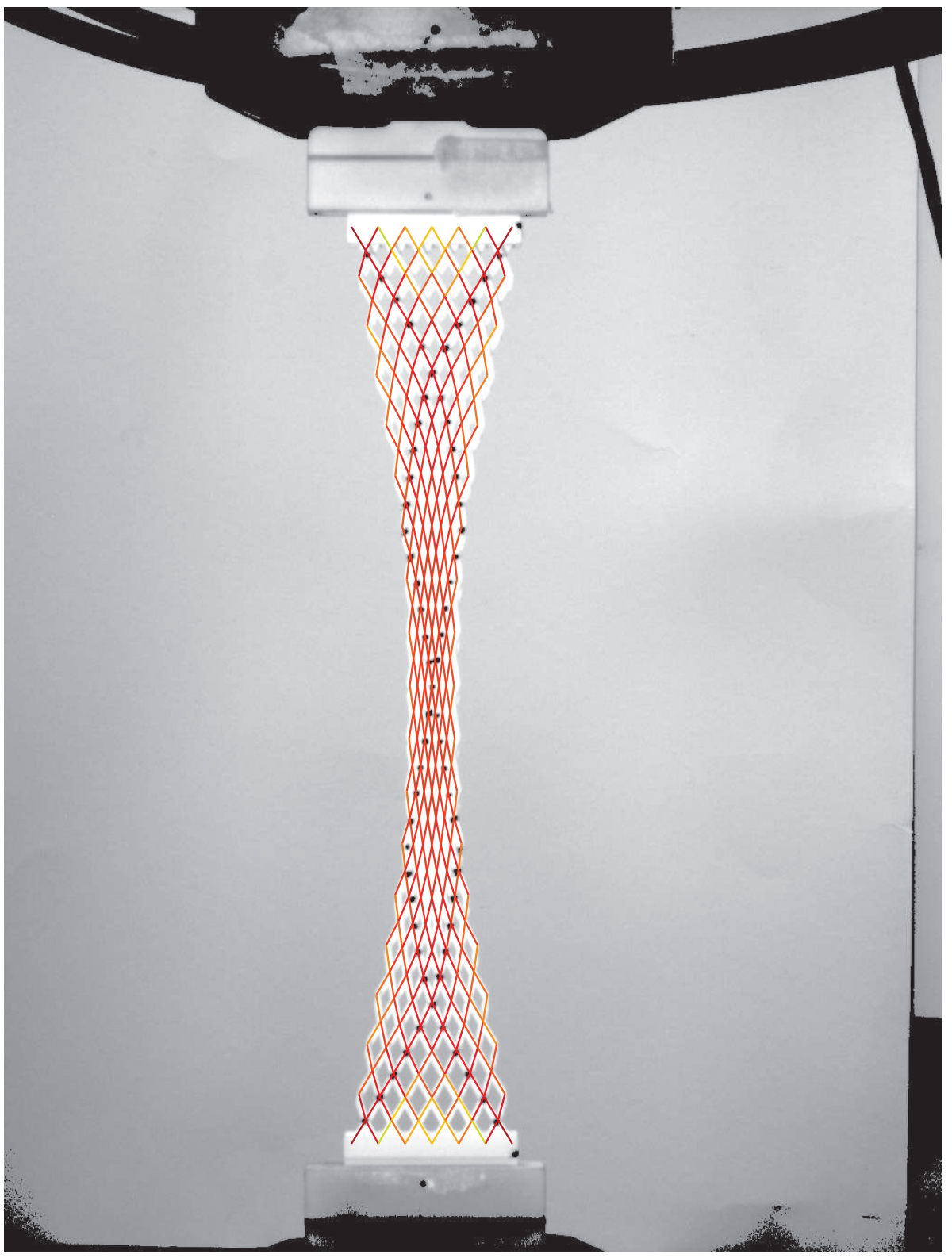

FIGURE 15. Pantographic lattice with non-orthogonal fibers $\left(\varphi=120^{\circ}\right)$ : overlapping of the final deformation for the experiment and the numerical simulation by using the Hencky-type numerical model. 


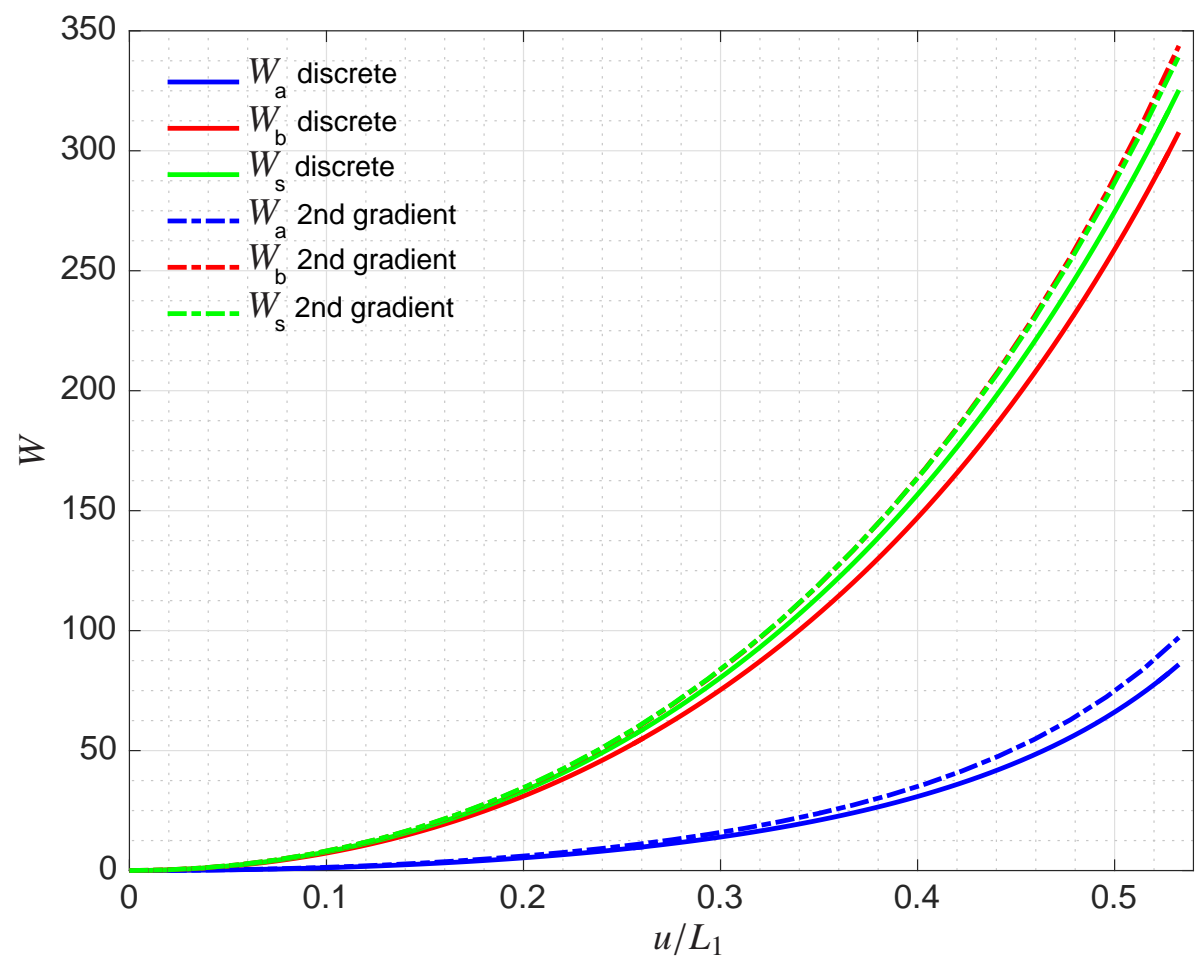

(a) Strain energy $W$ vs. $u / L_{1}$ distinct for axial (blue) bending (red) and shear (green) contribution for the discrete (continuous line) and 2nd gradient continuum (dot-dashed) model.

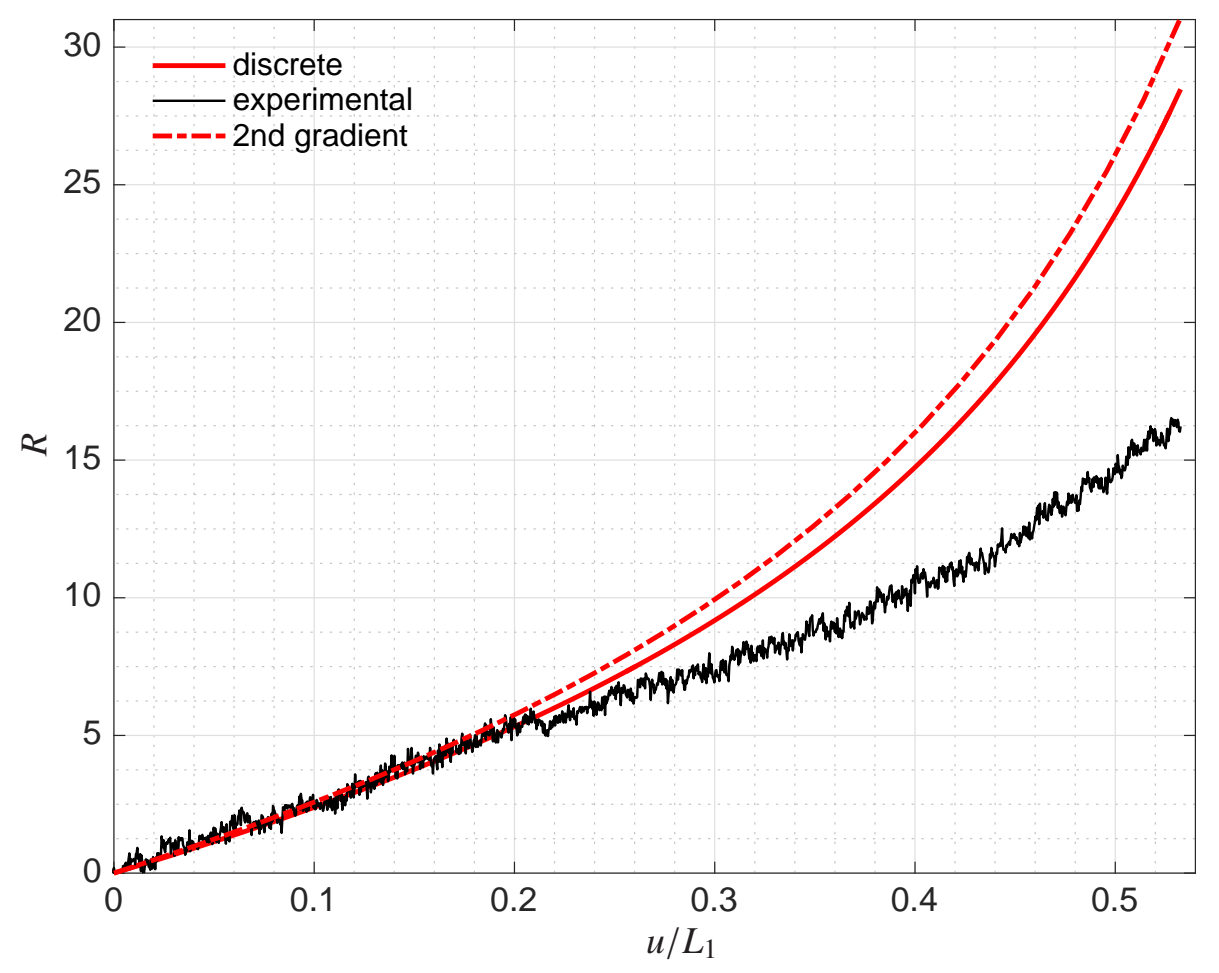

(b) Global structural reaction $R$ vs. $u / L_{1}$ for the discrete model (continuous red line), 2nd gradient continuum model (dot-dashed red line) and for the experiment (continuous black line)

FIgURe 16. Pantographic lattice with non-orthogonal fibres $\left(\varphi=60^{\circ}\right)$ : strain energy $W$ and global structural reaction $R$ for discrete and 2nd gradient continuum model. 


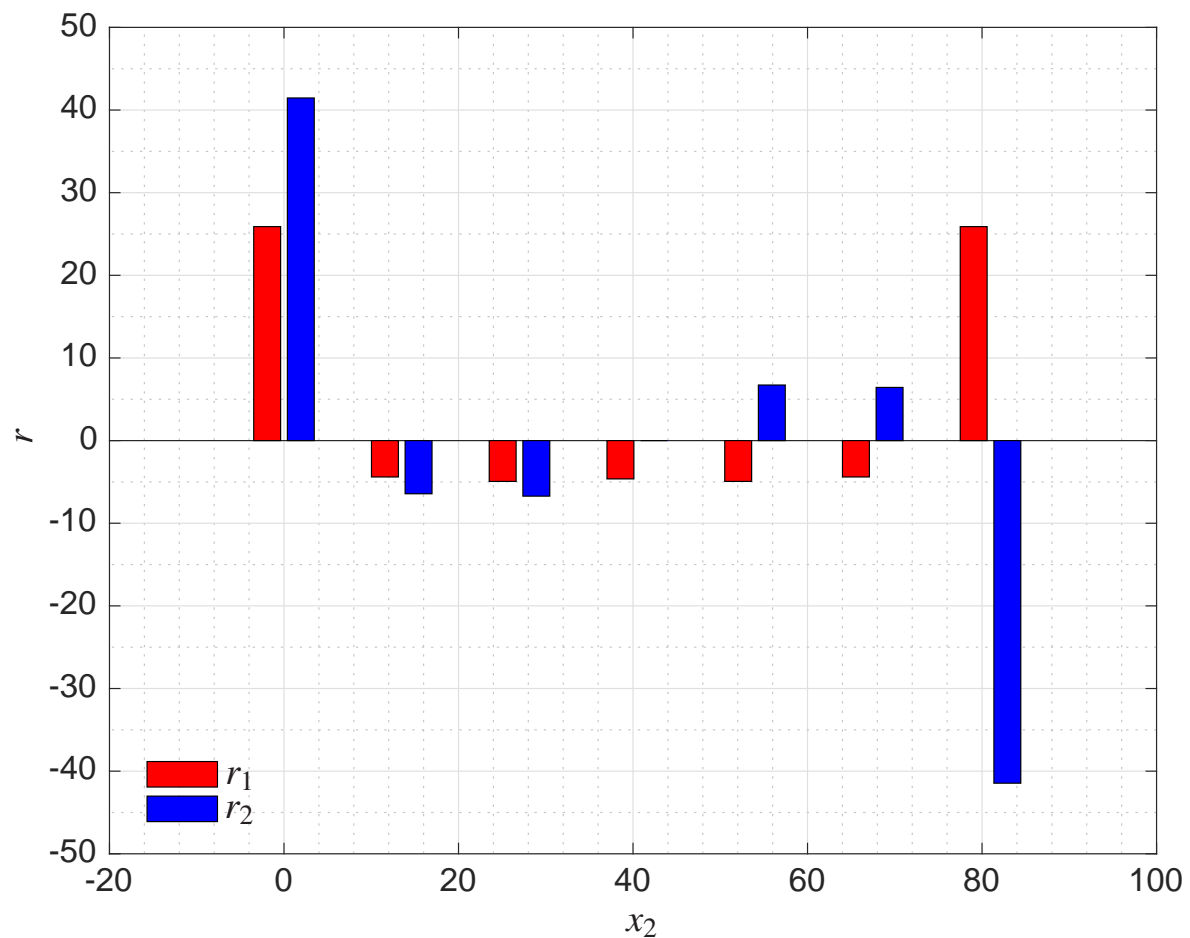

Figure 17. Pantograph lattice with non-orthogonal fibres $\left(\varphi=60^{\circ}\right)$ : density of structural reaction $r$ on the side $x_{1}=0$ for $u=u_{\max }$. 

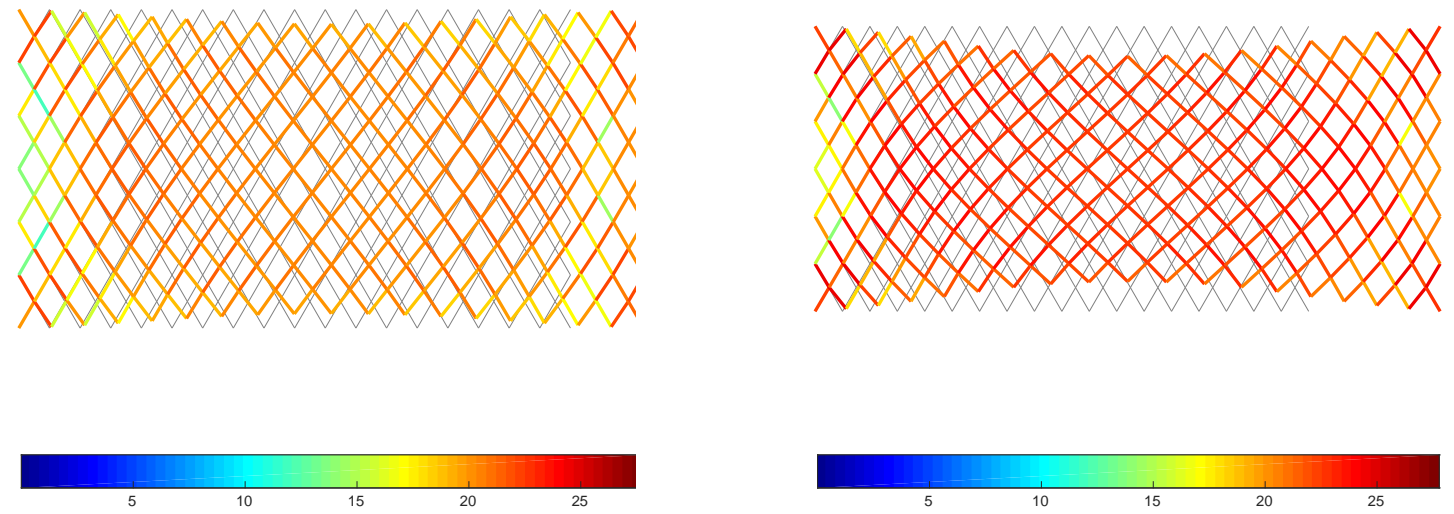

(a) $u / u_{\max }=0.25$

(b) $u / u_{\max }=0.5$
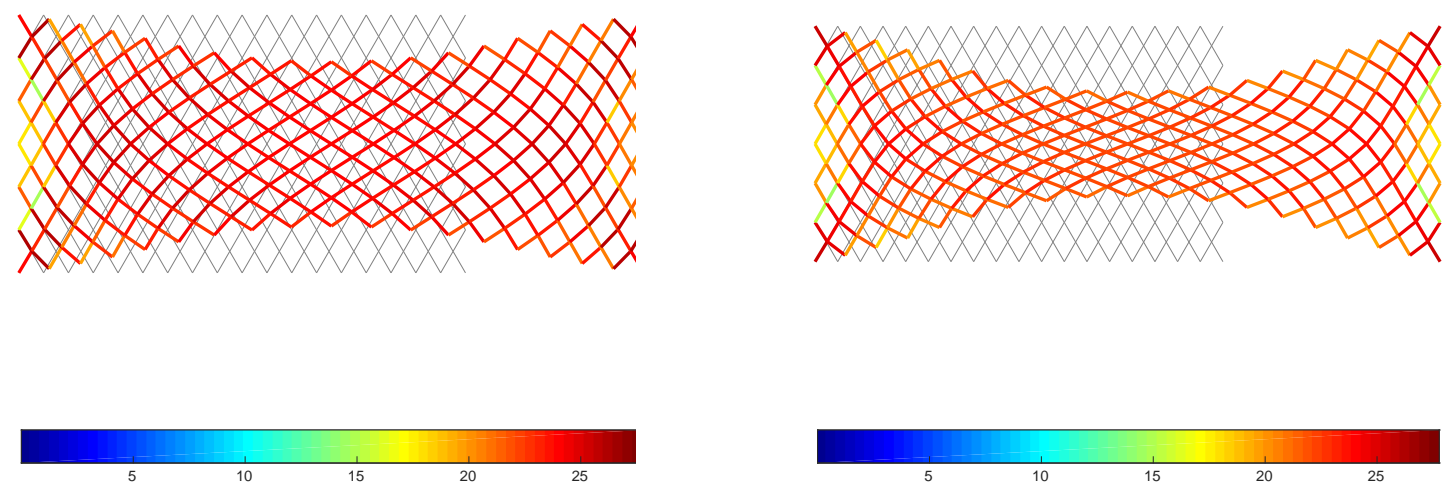

(c) $u / u_{\max }=0.75$

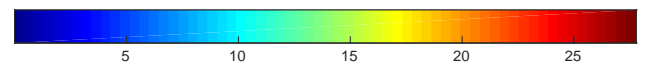

(d) $u / u_{\max }=1$

Figure 18. Pantographic lattice with non-orthogonal fibres $\left(\varphi=60^{\circ}\right)$ : history of deformation by using the Hencky-type numerical model (reference configuration is depicted in grey, colours indicate the strain energy density level). 


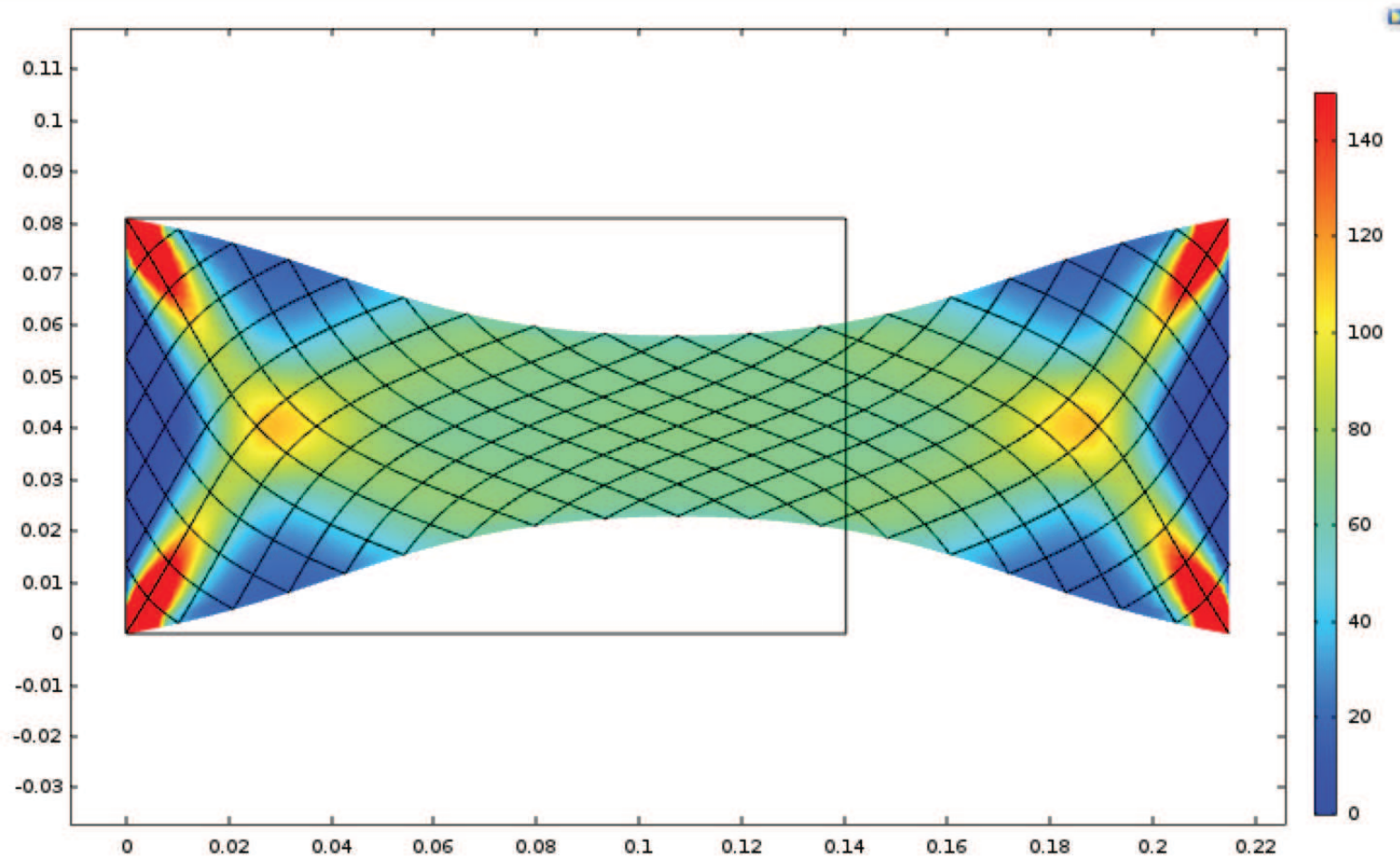

Figure 19. Pantograph lattice with non-orthogonal fibres $\left(\varphi=60^{\circ}\right)$ : deformation computed by the 2 nd gradient continuum model (colours represent the strain energy density level). 


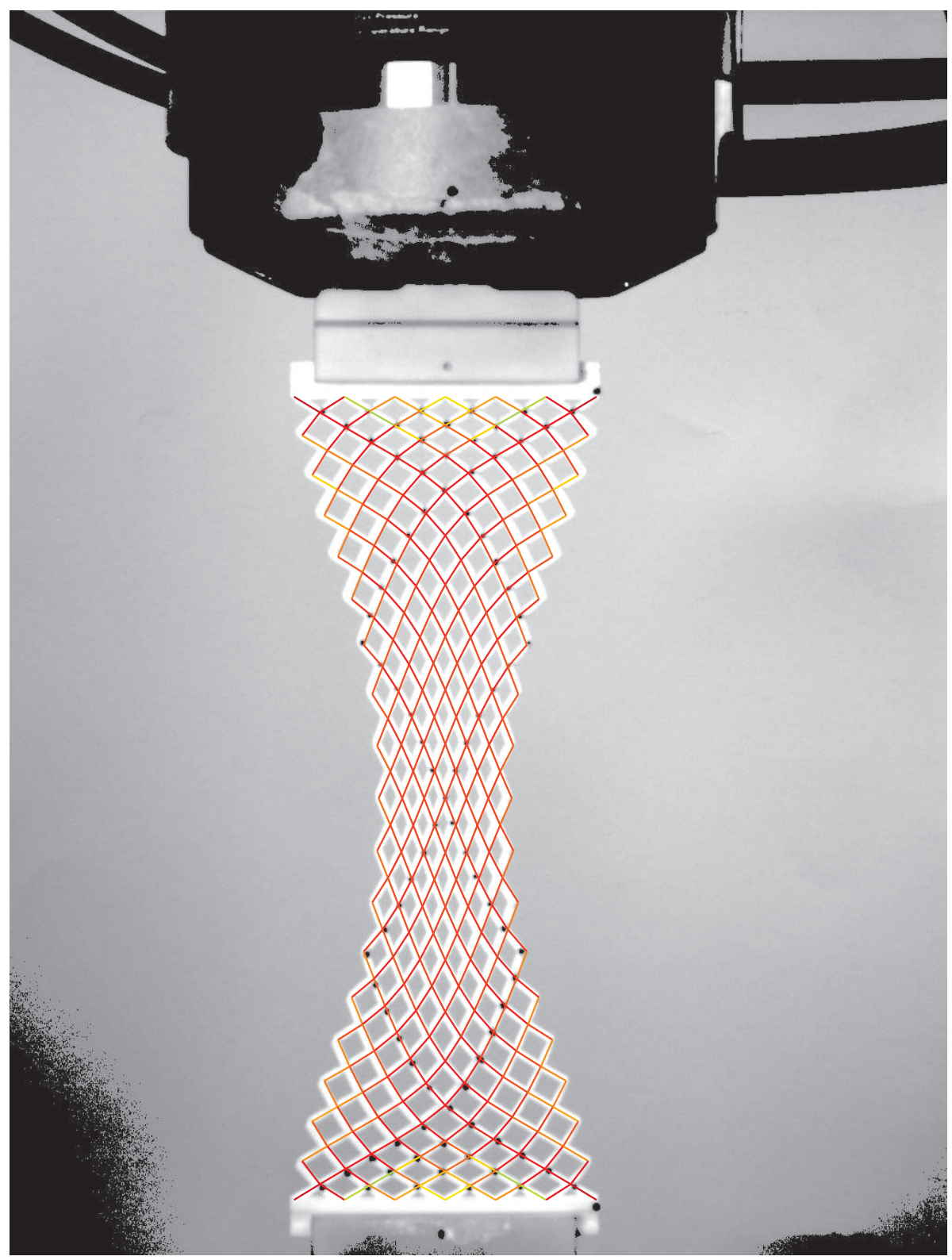

FiguRe 20. Pantographic lattice with non-orthogonal fibers $\left(\varphi=60^{\circ}\right)$ : overlapping of the final deformation for the experiment and the numerical simulation by using the Hencky-type numerical model. 